DRAFT VERSION NOVEMBER 5, 2018

Preprint typeset using LTEX style emulateapj v. 5/2/11

\title{
ANGULAR MOMENTUM TRANSPORT IN PROTOPLANETARY AND BLACK-HOLE ACCRETION DISKS: THE ROLE OF PARASITIC MODES IN THE SATURATION OF MHD TURBULENCE
}

\author{
MARTin E. PESSAH \\ Institute for Advanced Study, Princeton, NJ, 08540, USA; mpessah@ias.edu \\ Draft version November 5, 2018
}

\begin{abstract}
The magnetorotational instability (MRI) is considered a key process for driving efficient angular momentum transport in astrophysical disks. Understanding its non-linear saturation constitutes a fundamental problem in modern accretion disk theory. The large dynamical range in physical conditions in accretion disks makes it challenging to address this problem only with numerical simulations. We analyze the concept that (secondary) parasitic instabilities are responsible for the saturation of the MRI. Our approach enables us to explore dissipative regimes that are relevant to astrophysical and laboratory conditions that lie beyond the regime accessible to current numerical simulations. We calculate the spectrum and physical structure of parasitic modes that feed off the fastest, exact (primary) MRI mode when its amplitude is such that the fastest parasitic mode grows as fast as the MRI. We argue that this "saturation" amplitude provides an estimate of the magnetic field that can be generated by the MRI before the secondary instabilities suppress its growth significantly. Recent works suggest that the saturation amplitude of the MRI depends mainly on the magnetic Prandtl number. Our results suggest that, as long as viscous effects do not dominate the fluid dynamics, the saturation level of the MRI depends only on the Elsasser number $\Lambda_{\eta}$. We calculate the ratio between the stress and the magnetic energy density, $\alpha_{\mathrm{sat}} \beta_{\mathrm{sat}}$, associated with the primary MRI mode. We find that for $\Lambda_{\eta}>1$ Kelvin-Helmholtz modes are responsible for saturation and $\alpha_{\text {sat }} \beta_{\text {sat }}=0.4$, while for $\Lambda_{\eta}<1$ tearing modes prevail and $\alpha_{\text {sat }} \beta_{\text {sat }} \simeq 0.5 \Lambda_{\eta}$. Several features of numerical simulations designed to address the saturation of the MRI in accretion disks surrounding young stars and compact objects can be interpreted in terms of our findings.
\end{abstract}

Subject headings: accretion, accretion disks — black hole physics — instabilities — MHD — turbulence

\section{INTRODUCTION}

The transport of mass and angular momentum in accretion disks remains one of the least understood processes in modern astrophysics. The standard accretion disk model (Shakura \& Sunyaev 1973; Lynden-Bell \& Pringle 1974; Frank et al. 2002) is based on the assumption that turbulence provides an efficient mechanism for enabling accretion but magnetic fields, thought to be crucial for driving the turbulence, do not play an explicit role. It is currently believed that the magnetorotational instability (MRI; Velikhov 1959; Chandrasekhar 1960; Balbus \& Hawley 1991, 1998) is responsible for driving the magnetohydrodynamic (MHD) turbulence required for efficient angular momentum transport in astrophysical disks. However, at present, there are no accretion disk models that incorporate the physics driving angular momentum transport in a self-consistent way.

Since the appreciation of the relevance of the MRI to accretion disks, significant progress has been made in understanding the physics of the instability and characterizing the ensuing turbulent state. A large set of numerical simulations (see, e.g., Hawley et al. 1995; Brandenburg et al. 1995; Miller \& Stone 2000; Fleming et al. 2000; Sano \& Inutsuka 2001; Sano et al. 1998, 2004; Turner et al. 2007; Fromang et al. 2007; Lesur \& Longaretti 2007; Obergaulinger et al. 2009) have provided insight into the turbulent MHD flows under various physical conditions. However, the fundamental processes that determine the strength of the turbulence in the non-linear regime are yet to be deciphered. At present there have only been a handful of studies addressing the theoretical aspects of this problem (see, e.g., Goodman \& Xu 1994; Knobloch \& Julien 2005; Umurhan et al. 2007a.b; Tatsuno \& Dorland 2008;
Jamroz et al. 2008a b; Latter et al. 2009; Vishniac 2009; Pessah \& Goodman 2009). Thus, understanding the mechanisms that lead to the saturation of the MRI constitutes a fundamental problem in modern accretion physics.

Building models that capture the physics of the MRI and its saturation is crucial for constructing angular momentum transport models, and global disk models, beyond the standard viscous accretion disk (see, e.g., Kato \& Yoshizawa 1995; Ogilvie 2003; Pessah et al. 2006b). The identification, and eventual understanding, of correlations and scaling laws born out of the synergy between numerical simulations, analytical, and semi-analytical work (Pessah et al. 2006a, 2007, 2008; Blackman et al. 2008; Hubbard \& Blackman 2008; Lesaffre et al. 2009) provides important insight toward this goal. This could also provide a fruitful approach toward building sub-grid models for related microphysical processes which are unfeasible to incorporate in global simulations in a direct way (see, e.g., Arnett et al. 2009).

Pessah \& Goodman (2009) provided a summary of a parametric study of incompressible, MRI-parasitic instabilities in dissipative regimes accessible to current numerical simulations. They stated that the fastest growing modes are related to Kelvin-Helmholtz and tearing mode instabilities. In this work, we provide the details of the solutions to the differential equations involved and solve for the fastest growing modes in order to provide support for these assertions. We identify the existence of a critical Elsasser number of order unity and show that Kelvin-Helmholtz and tearing modes dominate in the quasi-ideal and resistive MHD regimes, respectively. By means of a systematic study of the parameter space involved, we reveal scaling laws that describe the behavior of the fastest growing parasites in these limits. The joint analysis of the asymptotic behav- 


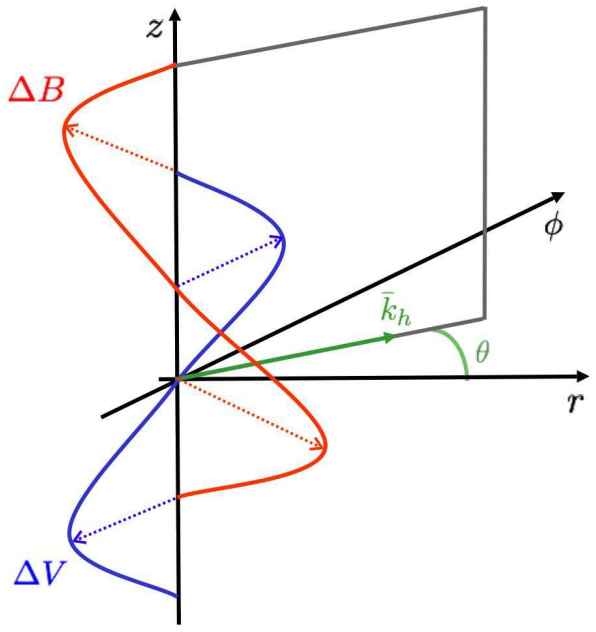

FIG. 1.- Three-dimensional representation of a primary MRI mode. The perturbations over the background Keplerian velocity and magnetic fields are given by $\Delta v=V_{0}\left(\cos \theta_{\mathrm{V}}, \sin \theta_{\mathrm{V}}, 0\right) \sin (K z)$ and $\Delta B=$ $B_{0}\left(\cos \theta_{\mathrm{B}}, \sin \theta_{\mathrm{B}}, 0\right) \cos (K z)$, respectively. For a given primary MRI mode with wavenumber $K$, the orientation of the planes containing $\Delta v$ and $\Delta B$ and the ratio of the amplitudes $V_{0} / B_{0}$ are functions of the viscosity and resistivity. The wavevector $\boldsymbol{k}_{\mathrm{h}}$ characterizes the horizontal wavelength of a given parasitic mode and $\theta$ denotes the angle between this vector and the radial direction, see also Fig. 2

ior of the MRI and the parasitic modes provides insight into the characteristics of a viable saturation mechanism in regimes that are relevant to astrophysical (see, e.g. Jin 1996; Gammie 1996; Sano \& Mivama 1999; Salmeron \& Wardle 2005; Brandenburg \& Subramanian 2005; Balbus \& Henri 2008) as well as experimental environments (see, e.g. Ji et al. 2001; Goodman \& Ji 2002; Sisan et al. 2004; Liu et al. 2006; Rüdiger et al. 2003).

\section{GENERAL CONSIDERATIONS AND PRIMARY MRI MODES}

Consider a cylindrical background characterized by an angular velocity profile $\Omega=\Omega(r) \check{z}$, threaded by a vertical magnetic field $\overline{\boldsymbol{B}}=\bar{B}_{z} \check{\boldsymbol{z}}$. We work in the incompressible limit which is relevant when the magnetic fields involved are so weak that the saturation of the MRI occurs at magnetic energies that are small compared to the thermal energy. We consider non-ideal effects due to a kinematic viscosity $\nu$ and resistivity $\eta$, both of which are assumed to be constant. The equations governing the local dynamics of this MHD fluid in the shearing box approximation are given by

$$
\begin{aligned}
\frac{\partial \boldsymbol{v}}{\partial t}+(\boldsymbol{v} \cdot \boldsymbol{\nabla}) \boldsymbol{v} & =-2 \boldsymbol{\Omega}_{0} \times \boldsymbol{v}+q \Omega_{0}^{2} \boldsymbol{\nabla}\left(r-r_{0}\right)^{2} \\
& -\frac{1}{\rho} \boldsymbol{\nabla}\left(P+\frac{\boldsymbol{B}^{2}}{8 \pi}\right)+\frac{(\boldsymbol{B} \cdot \boldsymbol{\nabla}) \boldsymbol{B}}{4 \pi \rho}+\nu \boldsymbol{\nabla}^{2} \boldsymbol{v},
\end{aligned}
$$

$\frac{\partial \boldsymbol{B}}{\partial t}+(\boldsymbol{v} \cdot \boldsymbol{\nabla}) \boldsymbol{B}=(\boldsymbol{B} \cdot \boldsymbol{\nabla}) \boldsymbol{v}+\eta \boldsymbol{\nabla}^{2} \boldsymbol{B}$,

where $P$ is the pressure, $\rho$ is the density, and the factor

$$
q \equiv-\left.\frac{d \ln \Omega}{d \ln r}\right|_{r_{0}},
$$

parametrizes the magnitude of the local shear at the fiducial radius $r_{0}$. The continuity equation reduces to $\boldsymbol{\nabla} \cdot \boldsymbol{v}=0$ and

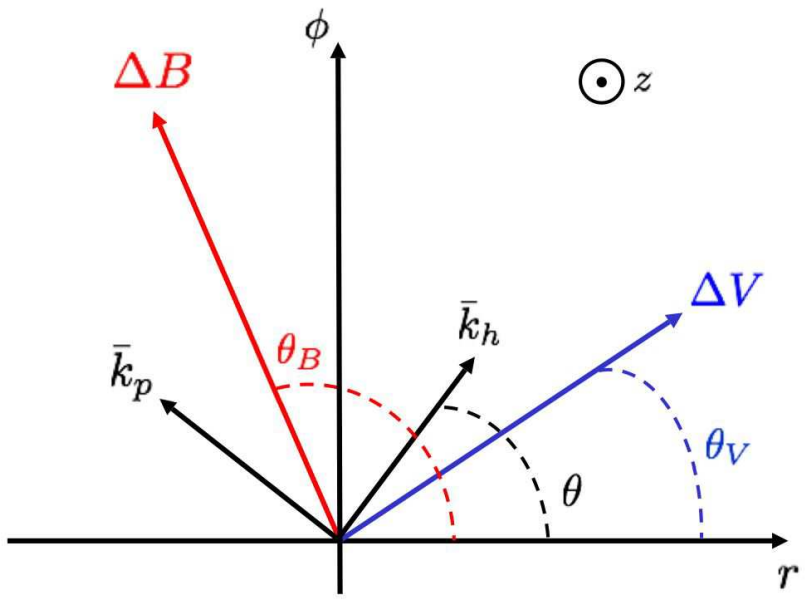

FIG. 2. - Two-dimensional representation of the projection of a primary MRI mode onto the $(\check{r}, \check{\phi})$ plane. The vectors $\boldsymbol{V}_{0}=V_{0}\left(\cos \theta_{\mathrm{V}}, \sin \theta_{\mathrm{V}}, 0\right)$ and $\boldsymbol{B}_{0}=B_{0}\left(\cos \theta_{\mathrm{B}}, \sin \theta_{\mathrm{B}}, 0\right)$ represent the projection of the MRI velocity and magnetic fields, with associated direction angles $\theta_{\mathrm{V}}$ and $\theta_{\mathrm{B}}$. The versors $\breve{\boldsymbol{k}}_{\mathrm{h}}$ and $\breve{\boldsymbol{k}}_{\mathrm{p}}$ characterize, respectively, the directions parallel and perpendicular to the horizontal wavevector $\boldsymbol{k}_{\mathrm{h}}$ of a given parasitic mode.

there is no need for an equation of state since the pressure can be determined from this condition.

The set of Equations (1) and (2) has solutions of the form

$$
\begin{aligned}
\boldsymbol{v} & =-q \Omega_{0}\left(r-r_{0}\right) \check{\boldsymbol{\phi}}+\boldsymbol{V}_{0} \sin (K z) e^{\Gamma t}, \\
\boldsymbol{B} & =\bar{B}_{z} \check{\boldsymbol{z}}+\boldsymbol{B}_{0} \cos (K z) e^{\Gamma t} .
\end{aligned}
$$

For a given wavenumber $K$ perpendicular to the disk midplane, the growth rate $\Gamma$ satisfies the dispersion relation

$$
\left(K^{2} \bar{v}_{\mathrm{A} z}^{2}+\Gamma_{\nu} \Gamma_{\eta}\right)^{2}+\kappa^{2}\left(K^{2} \bar{v}_{\mathrm{A} z}^{2}+\Gamma_{\eta}^{2}\right)-4 K^{2} \bar{v}_{\mathrm{A} z}^{2} \Omega_{0}^{2}=0,
$$

where $\Gamma_{\nu} \equiv \Gamma+\nu K^{2}, \Gamma_{\eta} \equiv \Gamma+\eta K^{2}, \kappa \equiv \sqrt{2(2-q)} \Omega_{0}$ is the epicyclic frequency, $\bar{v}_{\mathrm{A} z} \equiv \bar{B}_{z} / \sqrt{4 \pi \rho}$ is the Alfvén speed, and $\Omega_{0}$ is the local Keplerian frequency. The ratio of the amplitudes $V_{0} / B_{0}$ of the vectors characterizing the MRI velocity and magnetic fields,

$$
\begin{aligned}
& \boldsymbol{V}_{0} \equiv V_{0}\left(\cos \theta_{\mathrm{V}}, \sin \theta_{\mathrm{V}}, 0\right), \\
& \boldsymbol{B}_{0} \equiv B_{0}\left(\cos \theta_{\mathrm{B}}, \sin \theta_{\mathrm{B}}, 0\right),
\end{aligned}
$$

as well as their directions $\theta_{\mathrm{V}}$ and $\theta_{\mathrm{B}}$, see Figures 1 and 2 , are known functions of $(\nu, \eta, K)$, see Pessah \& Chan (2008) for a detailed discussion. The growth rate $\Gamma$ has a unique maximum, $\Gamma_{\max }(\nu, \eta)$, at $K=K_{\max }(\nu, \eta)$.

Unless otherwise mentioned, we work with dimensionless variables defined in terms of the characteristic length and time scales set by the background Alfvén speed and the local angular frequency: $L_{0} \equiv \bar{v}_{\mathrm{A} z} / \Omega_{0}$ and $T_{0} \equiv 1 / \Omega_{0}$. We subsume the effects related to viscosity and resistivity into the dimensionless quantities

$$
\begin{aligned}
& \Lambda_{\nu} \equiv \frac{\bar{v}_{\mathrm{A} z}^{2}}{\nu \Omega_{0}}, \\
& \Lambda_{\eta} \equiv \frac{\bar{v}_{\mathrm{A} z}^{2}}{\eta \Omega_{0}},
\end{aligned}
$$

whose ratio is the magnetic Prandtl number, $\mathrm{Pm} \equiv \nu / \eta \equiv$ $\Lambda_{\eta} / \Lambda_{\nu}$. The quantity $\Lambda_{\eta}$ is known as the Elsasser number, while its viscous counterpart $\Lambda_{\nu}$ is related to the Reynolds number. In our dimensionless variables, magnetic field 
strengths are defined relative to the background field $\bar{B}_{z}$, while $\Lambda_{\nu} \equiv \nu^{-1}$ and $\Lambda_{\eta} \equiv \eta^{-1}$.

\section{STABILITY OF MRI MODES}

The exact equations for the dynamical evolution of the velocity and magnetic fields corresponding to secondary instabilities $\delta \boldsymbol{v}(\boldsymbol{x}, t)$ and $\delta \boldsymbol{B}(\boldsymbol{x}, t)$ affecting an MRI mode are obtained by substituting in Equations (1) and (2) the ansatz

$$
\begin{aligned}
\boldsymbol{v} & =-q \Omega_{0}\left(r-r_{0}\right) \check{\boldsymbol{\phi}}+\boldsymbol{V}_{0} \sin (K z) e^{\Gamma t}+\delta \boldsymbol{v}, \\
\boldsymbol{B} & =\bar{B}_{z} \check{\boldsymbol{z}}+\boldsymbol{B}_{0} \cos (K z) e^{\Gamma t}+\delta \boldsymbol{B} .
\end{aligned}
$$

This procedure leads to a set of partial differential equations in space and time whose solution is beyond the scope of the present paper. It is possible, however, to gain insight into the growth rates and physical properties of the secondary instabilities by adopting some simplifying assumptions.

\subsection{Assumptions}

In order to make the problem of the stability of the non-ideal MRI modes against parasitic instabilities more tractable we adopt the same set of assumptions and approximations stated in Pessah \& Goodman (2009), see also Latter et al. (2009), which are the same as those adopted by Goodman \& Xu (1994) for ideal magnetohydrodynamics (MHD). The central simplification reduces to assuming that the exact (primary) MRI modes can be considered as a timeindependent background from which the (secondary) parasitic modes feed off. Because the growth rates of the parasitic modes increase as the amplitude of the primary mode increases, this approximation is better satisfied when the amplitude of the MRI modes is large compared to the background vertical field. In this case, we can also neglect the influence of the weak vertical background field, the Coriolis force, and the background shear flow on the dynamics of the secondary modes.

There is a subtlety associated with this assumption that is worth stating explicitly. The amplitude of the MRI cannot be assumed to be arbitrarily large. If this were the case, the parasites would drain an amount of energy comparable to the energy of the primary mode exceedingly fast. This would call into question whether the primary mode would have been able to reach the assumed amplitude in the first place. There exists a regime in which the amplitude of the primary modes is large enough that they can be taken as time-independent, but no so large that the fast-growing secondaries would have prevented the MRI from reaching the assumed amplitude.

Here, we are interested in estimating the amplitude to which the MRI can grow to in order for the fastest parasitic modes to have growth rates that are comparable to that of the primary mode upon which they feed. The motivation to calculate this "saturation" amplitude, i.e., $B_{0}^{\text {sat }}$, is that the parasites will be able to drain an amount of energy of order $\left(B_{0}^{\text {sat }}\right)^{2}$ from the primary modes shortly after their growth rates are comparable to that of the primary modes. We stress that the value of $B_{0}$ at which the amplitude of the parasites is similar to that of the primary MRI mode upon which they feed, is estimated to be a factor of a few larger that $B_{0}^{\text {sat }}$, see the discussion in Pessah \& Goodman (2009). Thus, it does not seem consistent to assume ab initio that the MRI modes can reach amplitudes $B_{0} \gg B_{0}^{\text {sat }}$. The price that we pay for working in the regime $B_{0} \simeq B_{0}^{\text {sat }}$ is that the assumption of a stationary background is only marginally satisfied.

\subsection{Equations of Motion for the Parasites}

Under the assumptions stated above, the equations governing the dynamics of the secondary instabilities are

$$
\begin{aligned}
\partial_{t} \delta \boldsymbol{v} & +(\Delta \boldsymbol{v} \cdot \boldsymbol{\nabla}) \delta \boldsymbol{v}+(\delta \boldsymbol{v} \cdot \boldsymbol{\nabla}) \Delta \boldsymbol{v}=-\boldsymbol{\nabla}(\delta P+\Delta \boldsymbol{B} \cdot \delta \boldsymbol{B}) \\
& +(\Delta \boldsymbol{B} \cdot \boldsymbol{\nabla}) \delta \boldsymbol{B}+(\delta \boldsymbol{B} \cdot \boldsymbol{\nabla}) \Delta \boldsymbol{B}+\nu \boldsymbol{\nabla}^{2} \delta \boldsymbol{v} \\
\partial_{t} \delta \boldsymbol{B}+ & (\Delta \boldsymbol{v} \cdot \boldsymbol{\nabla}) \delta \boldsymbol{B}+(\delta \boldsymbol{v} \cdot \boldsymbol{\nabla}) \Delta \boldsymbol{B}= \\
& \quad(\Delta \boldsymbol{B} \cdot \boldsymbol{\nabla}) \delta \boldsymbol{v}+(\delta \boldsymbol{B} \cdot \boldsymbol{\nabla}) \Delta \boldsymbol{v}+\eta \boldsymbol{\nabla}^{2} \delta \boldsymbol{B}
\end{aligned}
$$

where $\boldsymbol{\nabla} \cdot \delta \boldsymbol{v}=\boldsymbol{\nabla} \cdot \delta \boldsymbol{B}=0, \delta P$ stands for the pressure perturbation, and we have defined the time-independent amplitude of an unstable MRI mode with wavenumber $K$ as

$$
\begin{aligned}
\Delta \boldsymbol{v} & \equiv \boldsymbol{V}_{0} \sin (K z), \\
\Delta \boldsymbol{B} & \equiv \boldsymbol{B}_{0} \cos (K z) .
\end{aligned}
$$

We seek solutions to Equations (13) and (14) of the form

$$
\begin{aligned}
\delta \boldsymbol{v}(\boldsymbol{x}, t) & =\delta \boldsymbol{v}_{0}(z) \exp [s t-i \boldsymbol{k} \cdot \boldsymbol{x}], \\
\delta \boldsymbol{B}(\boldsymbol{x}, t) & =\delta \boldsymbol{B}_{0}(z) \exp [s t-i \boldsymbol{k} \cdot \boldsymbol{x}],
\end{aligned}
$$

Here, $\boldsymbol{k}=\boldsymbol{k}_{\mathrm{h}}+k_{z} \check{z}$, where $\boldsymbol{k}_{\mathrm{h}} \equiv k_{x} \check{x}+k_{y} \check{y} \equiv k_{\mathrm{h}}(\cos \theta \check{x}+$ $\sin \theta \check{y}$ ) is a horizontal wavevector, see Figures 1 and 2 , and $k_{z}$ is a parameter with $0 \leq k_{z} / K \leq 1 / 2$. The eigenvalue $s$ determines the temporal evolution of the parasitic mode and it must be solved for together with the amplitudes $\delta \boldsymbol{v}_{0}(z)$ and $\delta \boldsymbol{B}_{0}(z)$, which are $2 \pi / K$-periodic functions in $z$. The solutions (17) and (18) are periodic if $k_{z} / K$ is a rational number.

The set of six differential Equations (13) and (14) can in principle be solved for the secondary velocity and magnetic fields $(\delta P$ can be eliminated using $\boldsymbol{\nabla} \cdot \delta \boldsymbol{v}=0)$ by requiring that the "boundary conditions"

$$
\begin{aligned}
\delta \boldsymbol{v}(\boldsymbol{x}+2 \pi / K \check{\boldsymbol{z}}, t) & =\delta \boldsymbol{v}(\boldsymbol{x}, t) \exp \left(2 \pi i k_{z} / K\right), \\
\delta \boldsymbol{B}(\boldsymbol{x}+2 \pi / K \check{\boldsymbol{z}}, t) & =\delta \boldsymbol{B}(\boldsymbol{x}, t) \exp \left(2 \pi i k_{z} / K\right),
\end{aligned}
$$

be satisfied for all $z$. We follow an alternative procedure that leads to higher order differential equations for $\delta v_{z}$ and $\delta B_{z}$. Substituting expressions (17) and (18) into Equations (13) and (14), using the divergenceless nature of the perturbed fields, and eliminating the pressure perturbation between the horizontal and vertical components of Equation (13) we obtain

$$
\begin{gathered}
(s+\nu \mathcal{Q}) \mathcal{Q} \delta v_{z}-i\left(\boldsymbol{k}_{\mathrm{h}} \cdot \Delta \boldsymbol{v}\right)\left(\mathcal{Q}-K^{2}\right) \delta v_{z} \\
+i\left(\boldsymbol{k}_{\mathrm{h}} \cdot \Delta \boldsymbol{B}\right)\left(\mathcal{Q}-K^{2}\right) \delta B_{z}=0 \\
(s+\eta \mathcal{Q}) \delta B_{z}+i\left(\boldsymbol{k}_{\mathrm{h}} \cdot \Delta \boldsymbol{B}\right) \delta v_{z}-i\left(\boldsymbol{k}_{\mathrm{h}} \cdot \Delta \boldsymbol{v}\right) \delta B_{z}=0 .
\end{gathered}
$$

Here, we have used the explicit form of $\Delta \boldsymbol{v}$ and $\Delta \boldsymbol{B}$ from Equations (15) and (16), and defined the differential operator $\mathcal{Q}=k_{\mathrm{h}}^{2}-\partial_{z}^{2}$. These correspond to the equations of motion for the parasites presented in Pessah \& Goodman (2009).

Thus, the assumptions stated at the beginning of this Section allows us to reduce the problem of analyzing the stability of the exact MRI modes against secondary perturbations in terms of a set of linear, ordinary differential equations with periodic coefficients.

\subsection{The Eigenvalue Problem and Its Solution}

The set of Equations (21) and (22) can in principle be integrated along the $z$-coordinate subject to the boundary conditions (19) and (20). It is more convenient, however, to work 
in Fourier space and transform the differential equations into algebraic equations, by seeking solutions of the form

$$
\begin{aligned}
\delta v_{z}\left(z ; k_{z}\right) & =\sum_{n=-\infty}^{\infty} \alpha_{n} e^{-i\left(n K+k_{z}\right) z} e^{-i \boldsymbol{k}_{\mathrm{h}} \cdot \boldsymbol{x}}, \\
\delta B_{z}\left(z ; k_{z}\right) & =\sum_{n=-\infty}^{\infty} \beta_{n} e^{-i\left(n K+k_{z}\right) z} e^{-i \boldsymbol{k}_{\mathrm{h}} \cdot \boldsymbol{x}} .
\end{aligned}
$$

When the operator $\mathcal{Q}$ acts on the Fourier Series each individual terms incurs a factor $Q_{n} \equiv k_{\mathrm{h}}^{2}+\left(k_{z}+n K\right)^{2}$. Thus, the differential Equations (21) and (22) lead to recursion relations for the Fourier coefficients $\left\{\alpha_{n}\right\}$ and $\left\{\beta_{n}\right\}$ of the form

$$
\begin{aligned}
0 & =2\left(s+\nu Q_{n}\right) Q_{n} \alpha_{n} \\
& -i\left(\boldsymbol{k}_{\mathrm{h}} \cdot \boldsymbol{B}_{0}\right)\left[\left(Q_{n-1}-K^{2}\right) \beta_{n-1}+\left(Q_{n+1}-K^{2}\right) \beta_{n+1}\right] \\
& -\left(\boldsymbol{k}_{\mathrm{h}} \cdot \boldsymbol{V}_{0}\right)\left[\left(Q_{n-1}-K^{2}\right) \alpha_{n-1}-\left(Q_{n+1}-K^{2}\right) \alpha_{n+1}\right], \\
0 & =2\left(s+\eta Q_{n}\right) \beta_{n} \\
& -i\left(\boldsymbol{k}_{\mathrm{h}} \cdot \boldsymbol{B}_{0}\right)\left(\alpha_{n-1}+\alpha_{n+1}\right)-\left(\boldsymbol{k}_{\mathrm{h}} \cdot \boldsymbol{V}_{0}\right)\left(\beta_{n-1}-\beta_{n+1}\right) .
\end{aligned}
$$

It is convenient to use the natural scales provided by the primary MRI mode and rescale the variables: $Q_{n} \rightarrow Q_{n} / K^{2}$, $k_{\mathrm{h}} \rightarrow k_{\mathrm{h}} / K, \alpha_{n} \rightarrow \alpha_{n} / B_{0}, \beta_{n} \rightarrow \beta_{n} / B_{0}$. We thus obtain

$$
\begin{aligned}
\frac{s}{K B_{0}} \alpha_{n} & =-\frac{\nu K^{2}}{K B_{0}} Q_{n} \alpha_{n} \\
& +i \frac{\left(\boldsymbol{k}_{\mathrm{h}} \cdot \check{\boldsymbol{B}}_{0}\right)}{2 Q_{n}}\left[\left(Q_{n-1}-1\right) \beta_{n-1}+\left(Q_{n+1}-1\right) \beta_{n+1}\right] \\
& +\frac{\left(\boldsymbol{k}_{\mathrm{h}} \cdot \check{\boldsymbol{V}}_{0}\right)}{2 Q_{n}} \frac{V_{0}}{B_{0}}\left[\left(Q_{n-1}-1\right) \alpha_{n-1}-\left(Q_{n+1}-1\right) \alpha_{n+1}\right],
\end{aligned}
$$

$$
\begin{array}{r}
\frac{s}{K B_{0}} \beta_{n}=-\frac{\eta K^{2}}{K B_{0}} Q_{n} \beta_{n}+i \frac{\left(\boldsymbol{k}_{\mathrm{h}} \cdot \check{\boldsymbol{B}}_{0}\right)}{2}\left(\alpha_{n-1}+\alpha_{n+1}\right) \\
+\frac{\left(\boldsymbol{k}_{\mathrm{h}} \cdot \check{\boldsymbol{V}}_{0}\right)}{2} \frac{V_{0}}{B_{0}}\left(\beta_{n-1}-\beta_{n+1}\right),(28)
\end{array}
$$

where the versors $\check{\boldsymbol{V}}_{0} \equiv \boldsymbol{V}_{0} / V_{0}$ and $\check{\boldsymbol{B}}_{0} \equiv \boldsymbol{B}_{0} / B_{0}$ provide the direction of the MRI velocity and magnetic fields. Recall that the ratio of the velocity to magnetic field amplitudes, $V_{0} / B_{0}$, for the primary MRI mode is not an independent variable; it can be calculated for each set of values $(\nu, \eta, K)$ (Pessah \& Chan 2008). It is convenient to scale the Fourier coefficients using $B_{0}$, rather than $V_{0}$, because the ratio $V_{0} / B_{0}$ is proportional to $\Lambda_{\eta}$ for $\Lambda_{\eta} \ll 1$.

The system of coupled linear Equations (27) and (28), with $n=-\infty, \ldots, \infty$, can be written in matrix form as $M \boldsymbol{q}=s \boldsymbol{q}$, where $M$ is a band-diagonal, complex, non-Hermitian matrix and the components of the eigenvector $\boldsymbol{q}$ are defined according to $q_{n}=\alpha_{n / 2}$ for $n=2 m$ and $q_{n}=\beta_{n / 2+1}$ for $n=2 m+1$. Boundary conditions such that $\left(\alpha_{n}, \beta_{n}\right) \rightarrow 0$ must be imposed on the eigenvectors in order to ensure that the Fourier Series (23) and (24) converge as $|n| \rightarrow \infty$.

In practice, we set $\alpha_{\mathrm{N}}=\beta_{\mathrm{N}}=0$ for $|N| \geq N_{\max }$ and diagonalize the $2\left(2 N_{\max }+1\right) \times 2\left(2 N_{\max }+1\right)$ matrix $M$ for a given set of values $\left(\nu, \eta, K, B_{0}, k_{z}, \theta, k_{\mathrm{h}}\right)$. The algorithm employed to diagonalize the matrix $M$ is based on the LAPACK routine ZGEEVX, using the option of applying a balancing transformation to improve the conditioning of the eigenvalues and eigenvectors. We solve for the finite set of $2\left(2 N_{\max }+1\right)$ eigenvalues $\{s\}$ and eigenvectors $\{\boldsymbol{q}\}$ (if needed), taking increasingly large values of $N_{\max }$ until convergence to the desired accuracy is reached; $N_{\max }=30$ seems to do a very good job across the parameter space explored.

\section{PARASITIC INSTABILITIES AND SATURATION OF MRI MODES}

It may seem that the large number of independent variables involved could prevent us from obtaining a global understanding of the saturation of the MRI across the parameter space. This is not the case, however. We first present the results of a systematic calculation of the saturation amplitude of the fastest growing MRI modes, and their associated stresses, and show that they exhibit simple behaviors. In Section 5 we provide an explanation of these results by analyzing the behavior of the primary MRI modes and the parasitic modes responsible for their saturation in different regions of parameter space.

\subsection{Parameter Space of Interest}

Astrophysical disks, MRI laboratory experiments, and numerical simulations span a wide range of values in the parameter space defined by $\left(\Lambda_{\nu}, \Lambda_{\eta}\right)$, or equivalently $\left(\Lambda_{\nu}, \mathrm{Pm}\right)$ :

Astrophysical Disks: The temperature and density in an accretion disk vary by many orders of magnitude across its radius. Thus, the kinematic viscosity, $\nu$, and magnetic diffusivity, $\eta$, are expected to span a wide range of values. In most astrophysical environments, the kinematic viscosity is usually very small and thus $\Lambda_{\nu} \gg 1$. For a protoplanetary disk $\Lambda_{\eta}$ is a steep function of radius with $\Lambda_{\eta} \simeq$ $5 \times 10^{-11}(B / 0.1 \mathrm{G})^{2}(R / \mathrm{AU})^{37 / 4}$, where $R$ is the distance to the central star, see Sano \& Miyama (1999). Balbus \& Henri (2008) find that the magnetic Prandtl number for accretion disks around black holes decreases monotonically with increasing radius and lies in the range $\mathrm{Pm} \sim 10^{-3}-10^{3}$ for $R / R_{\mathrm{s}} \simeq 3-10^{3}$, where $R_{s}$ is the Schwarzschild radius.

Laboratory Experiments: Current Taylor-Couette experiments using liquid metals to realize the MRI in laboratories are characterized by physical parameters given by $\nu \sim$ $3 \times 10^{-3} \mathrm{~cm}^{2} / \mathrm{s}, \eta \sim 3 \times 10^{3} \mathrm{~cm}^{2} / \mathrm{s}, \rho \sim 6 \mathrm{gr} / \mathrm{cm}^{-3}, \Omega \sim 10$ $40 \mathrm{~Hz}$, and $\bar{B}_{z} \sim 1-4 \times 10^{3} \mathrm{G}$ (Nornberg et al. 2010). The value of $\Lambda_{\nu}$ can be varied by changing the strength of the background magnetic field or the rotation rate; characteristic values are of the order of $\Lambda_{\nu} \sim 10^{5}-10^{6}$. The magnetic Prandtl number is approximately constant with $\mathrm{Pm} \sim 10^{-6}$.

Numerical Simulations: There have been recent numerical simulations of the non-linear development of the MRI in the shearing-box approximation exploring the effects of explicit viscosity and resistivity. Using our definitions, the range of parameters explored correspond to $\Lambda_{\nu} \sim 1-100$ and $\mathrm{Pm} \sim 0.1-10$ in the three-dimensional simulations in Lesur \& Longaretti (2007) and $\Lambda_{\nu} \sim 0.01-10$ and $\mathrm{Pm} \sim$ $10^{-3}-10^{4}$ in the axisymmetric simulations in Masada \& Sano (2008).

Motivated by previous studies that suggest that the saturation amplitude of the MRI is sensitive to the magnetic Prandtl number, see e.g., Umurhan et al. (2007a b) and Lesur \& Longaretti (2007); we consider $\left(\Lambda_{\nu}, \mathrm{Pm}\right)$ as independent parameters. In order to explore in a systematic way the large region of parameter space relevant to the various environments mentioned above, we consider the range of pa- 


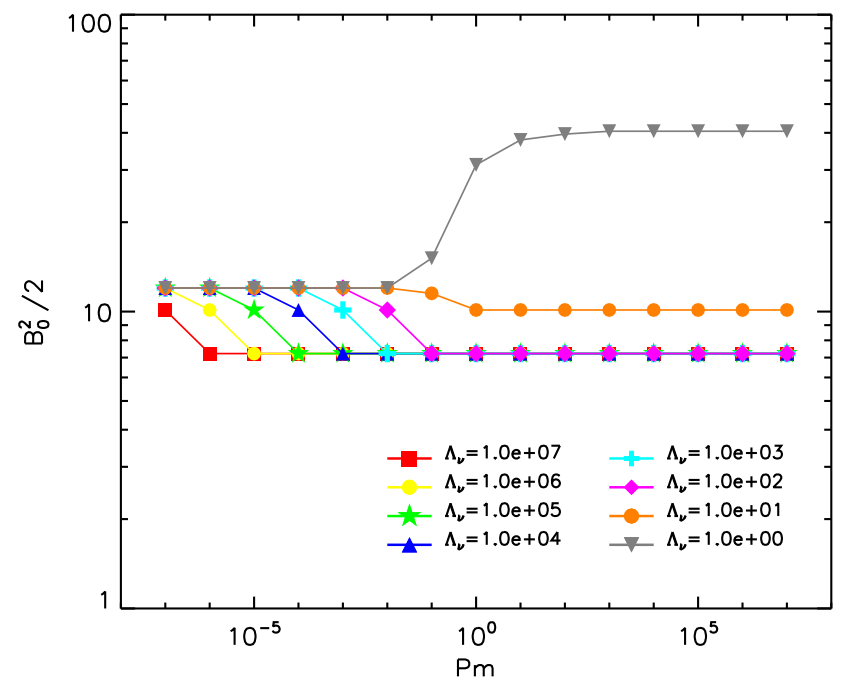

FIG. 3.- Magnetic energy density corresponding to the fastest growing primary MRI mode when the growth rate of the fastest parasitic mode matches $\Gamma_{\max }(\nu, \eta)$, i.e., $B_{0}=B_{0}^{\text {sat }}$. For $\Lambda_{\nu} \gtrsim 10$, there are two clear asymptotic regimes that correspond to $\operatorname{Pm} \Lambda_{\nu} \cong \Lambda_{\eta}$ larger or smaller than unity. The corresponding modes are associated with Kelvin-Helmholtz and tearing modes respectively, see Section 5

rameters defined by

$$
\begin{aligned}
\Lambda_{\nu} & =\left\{1,10, \ldots, 10^{7}\right\} \\
\operatorname{Pm} & =\left\{10^{-7}, 10^{-6}, \ldots, 10^{7}\right\} .
\end{aligned}
$$

This range of values is large enough to capture all the interesting behaviors and allows us to derive asymptotic scalings that are applicable outside this region of parameter space.

\subsection{Magnetic Energy Density and MRI-Stresses}

For each pair of values $\left(\Lambda_{\nu}, \mathrm{Pm}\right)$, we analyze the stability of the fastest growing primary MRI mode $K_{\max }(\nu, \eta)$ by solving the system of Equations (27) and (28) as a function of the variables $\left(B_{0}, k_{z}, \theta, k_{\mathrm{h}}\right)$. In order to explore the parameter space thoroughly, and identify the fastest parasitic modes, we find the growth rate of the secondary modes for a grid of values given by $k_{z}=\{0.0,0.1, \ldots, 0.5\}, \theta=$ $\left\{0^{\circ}, 5^{\circ}, \ldots, 180^{\circ}\right\}$, and $k_{\mathrm{h}}=\{0.0,0.01, \ldots, 1.0\}$ !. We find the fastest growing parasitic mode for a fixed value of the primary MRI magnetic field $B_{0}$ and iterate this procedure increasing $B_{0}$ until the fastest secondary instability matches the growth rate of the fastest MRI mode, i.e., $s_{\max }\left(\nu, \eta, K_{\max }\right)=$ $\Gamma_{\max }(\nu, \eta)$. This corresponds to the value $B_{0}^{\text {sat }}(\nu, \eta)$ that we denominate the "saturation" amplitude of the MRI magnetic field (Pessah \& Goodman 2009), which is shown in Figure 3.

For values $\Lambda_{\nu} \gtrsim 10$, the amplitude $B_{0}^{\text {sat }}$ displays two clear asymptotic behaviors, i.e., $\left(B_{0}^{\text {sat }}\right)^{2} / 2 \simeq 7$ and $\left(B_{0}^{\text {sat }}\right)^{2} / 2 \simeq$ 12 , depending on the magnetic Prandtl number. The 'critical' values for this transition, $\mathrm{Pm}^{\mathrm{c}}$, depend on $\Lambda_{\nu}$ and satisfy

$$
\operatorname{Pm}^{\mathrm{c}}\left(\Lambda_{\nu}\right) \Lambda_{\nu} \equiv \Lambda_{\eta}^{\mathrm{c}} \simeq 1 \text { for } \Lambda_{\nu} \gtrsim 10 .
$$

Therefore, for $\Lambda_{\nu} \gtrsim 10$, there is a critical Elsasser number of order unity that distinguishes two different regimes characterized by different saturation amplitudes, $B_{0}^{\text {sat }}$. For $\Lambda_{\nu}=\{1,10\}$, the MRI can reach amplitudes $\left(B_{0}^{\text {sat }}\right)^{2} / 2 \simeq 40$ for a wide range of magnetic Prandtl numbers Pm $\gtrsim 1$. This

\footnotetext{
1 The primary MRI modes seem to be always stable for $k_{\mathrm{h}}>1$.
}

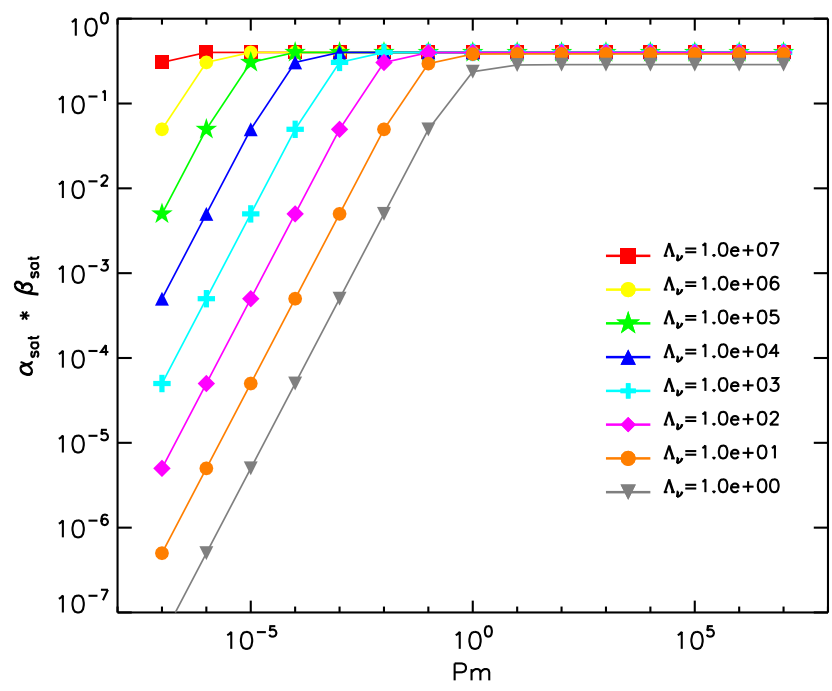

FIG. 4.- Predicted values for the product $\alpha_{\text {sat }} \beta_{\text {sat }}$ if saturation occurs when the fastest parasitic and primary MRI growth rates match. In the limit, $\Lambda_{\nu}, \mathrm{Pm} \gg 1, \alpha_{\text {sat }} \beta_{\text {sat }} \rightarrow 0.4$. In the inviscid, resistive limit, i.e., $\Lambda_{\nu} \gg 1$ and $\mathrm{Pm} \ll 1, \alpha_{\text {sat }} \beta_{\text {sat }} \rightarrow 0.5 \mathrm{Pm} \Lambda_{\nu}$. Despite the fact that the magnetic field at saturation asymptotes to a constant value, see Fig. 3 the dimensionless stress decreases linearly with $\operatorname{Pm} \Lambda_{\nu} \equiv \Lambda_{\eta}$ for $\operatorname{Pm} \Lambda_{\nu} \equiv \Lambda_{\eta} \lesssim 1$.

behavior is due to the viscous quenching of Kelvin-Helmholtz modes discussed in Pessah \& Goodman (2009) for Pm $\gtrsim 1$.

The saturation amplitude $B_{0}^{\text {sat }}$ varies roughly by only one order of magnitude across the large parameter space that we consider and is thus fairly insensitive to the value of the dissipation coefficients. In particular, as resistivity increases, i.e., $\Lambda_{\eta} \lesssim 1$, the magnetic field that can be generated by the MRI reaches an asymptotic, constant value. The situation is quite different for the stresses, however.

The dimensionless stress at saturation is given by $\alpha_{\text {sat }} \equiv$ $\bar{T}_{r \phi}^{\text {sat }} /\left(L_{z} \Omega_{0}\right)^{2}$, where $\bar{T}_{r \phi}^{\text {sat }} \equiv \bar{R}_{r \phi}^{\text {sat }}-\bar{M}_{r \phi}^{\text {sat }}$, is the sum of the Reynolds and Maxwell stresses

$$
\begin{aligned}
\bar{R}_{r \phi}^{\mathrm{sat}} & \equiv \frac{1}{L_{z}} \int_{-L_{z} / 2}^{L_{z} / 2} V_{0, r}^{\mathrm{sat}}(z) V_{0, \phi}^{\mathrm{sat}}(z) d z, \\
\bar{M}_{r \phi}^{\mathrm{sat}} & \equiv \frac{1}{L_{z}} \int_{-L_{z} / 2}^{L_{z} / 2} B_{0, r}^{\mathrm{sat}}(z) B_{0, \phi}^{\mathrm{sat}}(z) d z .
\end{aligned}
$$

Integrating these expressions we obtain the dimensionless stress $\alpha_{\text {sat }}$ in terms of the parameter $\beta \equiv \Omega_{0}^{2} L_{z}^{2} / \bar{v}_{\mathrm{A} z}^{2}$,

$$
\alpha_{\mathrm{sat}}=\frac{1}{2 \beta}\left[\left(V_{0}^{\mathrm{sat}}\right)^{2} \sin \theta_{\mathrm{V}} \cos \theta_{\mathrm{V}}-\left(B_{0}^{\mathrm{sat}}\right)^{2} \sin \theta_{\mathrm{B}} \cos \theta_{\mathrm{B}}\right] .
$$

We can obtain an expression for the ratio between the stress and the magnetic energy density associated with the primary MRI mode by relating the initial value of the $\beta$-parameter, $\beta \propto 1 / \bar{B}_{z}^{2}$, with an estimate for its corresponding value at saturation, i.e., $\beta_{\text {sat }}=\beta / B_{0}^{2}$. This leads to

$$
\begin{aligned}
& \alpha_{\text {sat }} \beta_{\text {sat }}=\frac{1}{2}\left[\left(\frac{V_{0}^{\text {sat }}}{B_{0}^{\text {sat }}}\right)^{2} \sin \theta_{\mathrm{V}} \cos \theta_{\mathrm{V}}-\sin \theta_{\mathrm{B}} \cos \theta_{\mathrm{B}}\right] \\
& 2 \text { Pessah \& Goodman }(2009) \text { defined } \alpha_{\text {sat }} \equiv \bar{T}_{r \phi}^{\text {sat }} /\left(S L_{z}\right)^{2} \text { and } \\
& \beta \equiv S^{2} L_{z}^{2} / \bar{v}_{\Delta}^{2} \text {, with } S \equiv 3 \Omega_{0} / 2 \text {, in order to compare results with } \\
& \text { Lesur \& Longaretti (2007). The definitions adopted here do not affect the } \\
& \text { expression or the numerical value for the product } \alpha \beta \text {. }
\end{aligned}
$$



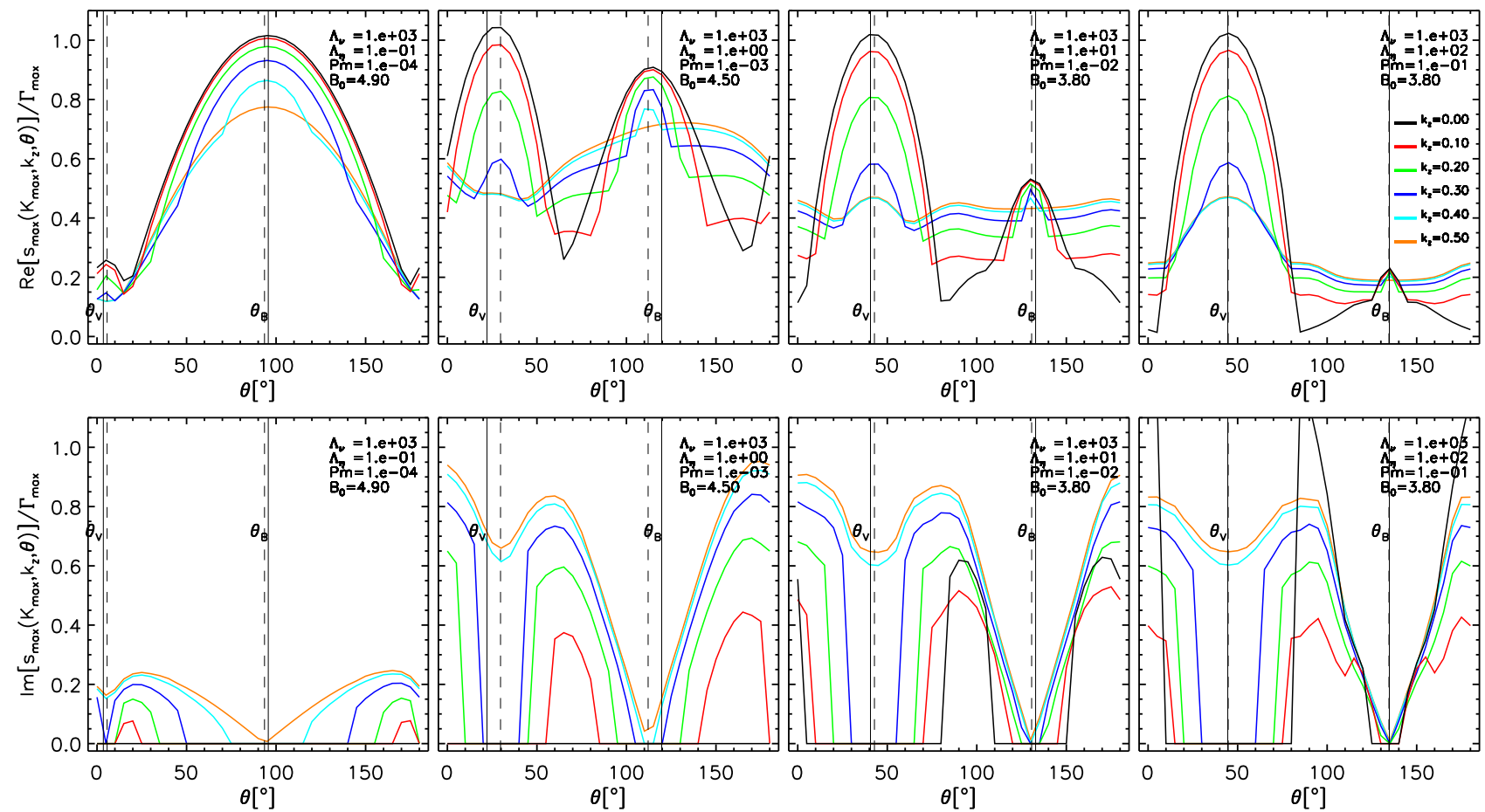

FIG. 5.- Real and imaginary parts for the fastest parasitic instabilities normalized by the growth rate of the fastest MRI mode as a function of the orientation of the horizontal wavevector $\boldsymbol{k}_{\mathrm{h}}$ with respect to the radial $(\theta=0)$ direction. The panels correspond to $\Lambda_{\eta}=\left\{0.1,1,10,10^{2}\right\}$, from left to right, with $\Lambda_{\nu} \gg 1$. The MRI magnetic field $B_{0}=B_{0}^{\mathrm{sat}}(\nu, \eta)$ is such that the fastest parasitic growth rate, maximized over $k_{\mathrm{h}}, \theta$, and $k_{z}$, matches the growth rate of the fastest primary MRI mode, $\Gamma_{\max }(\nu, \eta)$. The parasites present fastest growth along the directions $\theta_{\max } \approx \theta_{\mathrm{V}}$ and $\theta_{\max } \approx \theta_{\mathrm{B}}$, shown as solid lines, and they are associated with Kelvin-Helmholtz and tearing mode instabilities, respectively.

The dependence of the product $\alpha_{\text {sat }} \beta_{\text {sat }}$ on Pm for different values of $\Lambda_{\nu}$ is shown in Figure 4. The existence of two different asymptotic behaviors for $\Lambda_{\nu} \gtrsim 10$ is evident:

$$
\begin{array}{ll}
\alpha_{\text {sat }} \beta_{\text {sat }} \simeq 0.4 & \text { for } \quad \operatorname{Pm} \Lambda_{\nu}>1, \\
\alpha_{\text {sat }} \beta_{\text {sat }} \simeq 0.5 \operatorname{Pm} \Lambda_{\nu} & \text { for } \quad \operatorname{Pm} \Lambda_{\nu}<1 .
\end{array}
$$

The asymptotic behavior for $\Lambda_{\nu} \gg 1$ and $\mathrm{Pm} \gg 1$ corresponds to the ideal MHD limit with $\Lambda_{\eta} \gg \Lambda_{\nu} \gg 1$. It is worth mentioning that numerical simulations of MRI driven turbulence (with no explicit dissipation), carried out over a wide range of physical conditions, lead to saturation values for the parameters $\alpha_{\mathrm{sat}}$ and $\beta_{\mathrm{sat}}$ that vary by several orders of magnitude (see, e.g. Pessah et al. 2006a, and references therein). However, their product remains roughly constant with $\alpha_{\text {sat }} \beta_{\text {sat }} \simeq 0.5$ (Hawley et al. 1995; Sano et al. 2004). Despite the approximations that we adopted for calculating this quantity, the value in Equation (36) is remarkably close to the results obtained in numerical simulations. We note that Blackman et al. (2008) provide independent arguments, involving turbulent eddies, to support the idea that $\alpha_{\text {sat }}$ and $\beta_{\text {sat }}$ should be anti-correlated. They estimate that $\alpha_{\text {sat }} \beta_{\text {sat }} \simeq 0.7$, for an isothermal equation of state.

The product $\alpha_{\text {sat }} \beta_{\text {sat }}$ presents a very different behavior below a 'critical' value, $\operatorname{Pm}^{\mathrm{c}}\left(\Lambda_{\nu}\right) \Lambda_{\nu} \equiv \Lambda_{\eta}^{\mathrm{c}} \simeq 1$. In this regime, the stress decreases linearly with both Pm and $\Lambda_{\nu}$ for $\operatorname{Pm} \Lambda_{\nu} \lesssim 1$. The product $\operatorname{Pm} \Lambda_{\nu}$ corresponds of course to the Elsasser number, $\Lambda_{\eta}$. We write the expression for $\operatorname{Pm} \Lambda_{\nu}$ in Equation (37) explicitly because it is not a priori evident that the quantity $\alpha_{\text {sat }} \beta_{\text {sat }}$ should scale linearly with both Pm and $\Lambda_{\nu}$. For instance, it could have depended on some power of the magnetic Prandtl number, e.g., $\mathrm{Pm}^{1 / 2}$ with a weaker

dependence on $\Lambda_{\nu}$. This is indeed the type of dependence observed in the numerical simulations in Lesur \& Longaretti (2007), which address the regime $\Lambda_{\nu} \sim 1-100$ and $\mathrm{Pm} \sim$ $0.1-10$. However, in the regime $\Lambda_{\nu} \gtrsim 10$, our calculations lead to MRI stresses that depend only on the Elsasser number $\Lambda_{\eta}$ with $\bar{T}_{r \phi} \propto \Lambda_{\eta}$ for $\Lambda_{\eta} \lesssim 1$.

The existence of a critical Elsasser number of order unity is related to the fact that the fastest growing secondary modes are associated with Kelvin-Helmholtz instabilities for $\Lambda_{\eta}>1$ and tearing instabilities for $\Lambda_{\eta}<1$, provided that $\Lambda_{\nu} \gtrsim 10$. In order to provide support to these assertions, it is useful to examine the behavior of the growth rates of the parasitic modes as a function of the direction of the horizontal wavevector $\boldsymbol{k}_{\mathrm{h}}$, i.e., the angle $\theta$, see Figures 1 and 2 .

\subsection{Kelvin-Helmholtz vs. Tearing Modes}

It is of particular interest to understand which type of secondary modes are the first to reach growth rates that are comparable to the growth rate of the MRI in different regions of the parameter spaced spanned by the dissipation coefficients.

The upper and lower sets of panels in Figure 5 show the real and imaginary parts of the eigenvalues, $s_{\max }\left(\nu, \eta, K_{\max }, k_{z}, \theta\right)$, corresponding to the fastest growing parasitic modes that feed off the fastest primary MRI mode for $B_{0}=B_{0}^{\text {sat }}(\nu, \eta)$. The different panels in this figure correspond to $\Lambda_{\eta}=\left\{0.1,1,10^{1}, 10^{2}\right\}$, from left to right, with $\Lambda_{\nu}=10^{3}$. The various curves in each panel correspond to different values of the parameter $k_{z}$. The vertical solid lines show the angles $\theta_{\mathrm{V}}(\nu, \eta)$ and $\theta_{\mathrm{B}}(\nu, \eta)$ associated with the velocity and magnetic fields of the fastest MRI mode, see Figures 1 and 2 . The vertical dashed lines in each panel show the directions perpendicular to $\theta_{\mathrm{B}}$ (left) and $\theta_{\mathrm{V}}$ (right). The MRI velocity and magnetic fields are close to orthogonal, except 
TABLE 1

Characterization of Fastest MRI and Parasitic Modes

\begin{tabular}{cccrcccccc}
\hline \hline$\Lambda_{\eta}$ & $\Gamma_{\max } / \Omega_{0}, s_{\max } / \Omega_{0}$ & $K_{\max }$ & $\theta_{\mathrm{V}}\left[^{\circ}\right]$ & $\theta_{\mathrm{B}}\left[^{\circ}\right]$ & $\theta_{\max }\left[^{\circ}\right]$ & $k_{\mathrm{h}, \max } / K_{\max }$ & $B_{0}^{\text {sat }} / \bar{B}_{z}$ & $V_{0} / B_{0}$ & Type \\
\hline $1 . \mathrm{E}-03$ & $7.50 \mathrm{E}-04$ & $8.66 \mathrm{E}-04$ & 0.0 & 90.1 & 90.0 & 0.48 & 4.9 & 0.0017 & $\mathrm{TM}$ \\
$1 . \mathrm{E}-02$ & $7.50 \mathrm{E}-03$ & $8.66 \mathrm{E}-03$ & 0.0 & 90.6 & 90.0 & 0.48 & 4.9 & 0.0173 & $\mathrm{TM}$ \\
$1 . \mathrm{E}-01$ & $7.39 \mathrm{E}-02$ & $8.55 \mathrm{E}-02$ & 3.5 & 95.6 & 95.0 & 0.48 & 4.9 & 0.1689 & $\mathrm{TM}$ \\
$1 . \mathrm{E}+00$ & $4.28 \mathrm{E}-01$ & $5.16 \mathrm{E}-01$ & 22.1 & 119.7 & 30.0 & 0.59 & 4.5 & 0.7187 & $\mathrm{KH}$ \\
$1 . \mathrm{E}+01$ & $6.96 \mathrm{E}-01$ & $8.88 \mathrm{E}-01$ & 40.6 & 132.9 & 40.0 & 0.59 & 3.8 & 0.7816 & $\mathrm{KH}$ \\
$1 . \mathrm{E}+02$ & $7.44 \mathrm{E}-01$ & $9.59 \mathrm{E}-01$ & 44.5 & 134.7 & 45.0 & 0.59 & 3.8 & 0.7751 & $\mathrm{KH}$ \\
$1 . \mathrm{E}+03$ & $7.49 \mathrm{E}-01$ & $9.67 \mathrm{E}-01$ & 45.0 & 135.0 & 45.0 & 0.59 & 3.8 & 0.7743 & $\mathrm{KH}$ \\
$1 . \mathrm{E}+04$ & $7.50 \mathrm{E}-01$ & $9.67 \mathrm{E}-01$ & 45.0 & 135.0 & 45.0 & 0.59 & 3.8 & 0.7742 & $\mathrm{KH}$ \\
\hline
\end{tabular}

NOTE. - Data corresponding to the fastest growing MRI modes and their associated fastest parasitic modes for $\Lambda_{\nu} \gg 1$. Note the asymptotic behaviors at low and high Elsasser numbers and the sharp transition around $\Lambda_{\eta} \simeq 1$. The resistive $\left(\Lambda_{\eta} \ll 1\right)$ and ideal $\left(\Lambda_{\eta} \gg 1\right)$ MHD regime are dominated by tearing modes (TM) and Kelvin-Helmholtz (KH) modes, respectively.

for $\Lambda_{\eta} \simeq 1$, where the directions parallel to $\theta_{\mathrm{V}}$ and perpendicular to $\theta_{\mathrm{B}}$ differ by a few degrees.

The fastest parasitic modes that determine the values of $B_{0}^{\text {sat }}$ shown in Figure 3 are non-axisymmetric $\left(\theta_{\max } \neq 0\right)$; their horizontal wavevectors, $\boldsymbol{k}_{\mathrm{h}, \max }$, are nearly aligned with either the velocity or the magnetic field of the primary mode. The first type reach their maximum growth rates for $\theta_{\max } \simeq$ $\theta_{\mathrm{V}}$, i.e., the direction of the MRI velocity field, $\Delta \boldsymbol{V}$. These correspond to Kelvin-Helmholtz modes and dominate for $\Lambda_{\eta} \geq 1$. The second type of modes grow the fastest along the direction $\theta_{\max } \simeq \theta_{\mathrm{B}}$, i.e., the direction of the MRI magnetic field, $\Delta \boldsymbol{B}$. These are tearing modes enabled by resistive reconnection of the MRI magnetic field. They dominate for $\Lambda_{\eta}<1$ and become increasingly relevant as $\Lambda_{\eta}$ decreases.

Independently of the value of the dissipation coefficients, the fastest growing Kelvin-Helmholtz and tearing modes have the same vertical periodicity as the primary mode, i.e., $k_{z}=$ 0 . As illustrated in the lower panels of Figure 5, the fastest growing Kelvin-Helmholtz modes have purely real growth rates for $k_{z} \lesssim 0.25$; while they have imaginary parts that are similar to their real parts for $k_{z} \gtrsim 0.25$. The latter correspond to the "Type-II" modes discussed in Goodman \& Xu (1994), see also Latter et al. (2009). These are clearly KelvinHelmholtz modes since they present their maximum growth along the direction of the MRI velocity field. These modes might be relevant in axisymmetric simulations of viscous, resistive MRI because they have growth rates that are comparable, or even larger, than the axisymmetric modes with $k_{z}=0$. The fastest growing tearing modes have zero, or very small, imaginary parts for every value of $k_{z}$.

It is clear from Figures 3, 4, and 5that there is a sharp transition in behavior around a critical Elsasser number of order unity. Table 1 provides information concerning the growth rates and geometric structure of both the fastest growing primary MRI mode and the associated fastest parasitic mode for a range of Elsasser numbers $\Lambda_{\eta}=\left\{10^{-3}, \ldots, 10^{4}\right\}$, with $\Lambda_{\nu}=10^{3}$. Note that the horizontal wavelength of the fastest growing modes, for both tearing and Kelvin-Helmholtz modes, is roughly a factor of two larger than the vertical wavelength of the primary mode upon which they feed. Tearing modes and Kelvin-Helmholtz modes present the fastest growth at low and high Elsasser numbers, respectively. These two regimes are characterized by asymptotic behaviors that are already evident for $\Lambda_{\eta} \simeq 10^{ \pm 2}$.

The existence of these scalings simplifies the characterization of the physical nature of the parasitic modes in different regions of parameter space. Thus, we first analyze the asymptotic regimes al large and low Elsasser number and postpone the explicit analysis of the physical structure of the eigenmodes until Section 6. It will then be evident that it is only necessary to understand the nature of the fastest modes with moderate Elsasser number $\Lambda_{\eta} \simeq 1$ and that the characteristics of the modes for $\Lambda_{\eta} \ll 1$ and $\Lambda_{\eta} \gg 1$ can be obtained using the scaling relations derived next.

\section{ASYMPTOTIC BEHAVIORS AND SCALING LAWS}

The regime where viscosity plays an important role, i.e., $\Lambda_{\nu} \lesssim 10$, was explored in detail in Pessah \& Goodman (2009). Hereafter, we focus our attention on the regime where $\Lambda_{\nu} \geq 10^{2}$, i.e., $\Lambda_{\nu} \gg 1$, which is relevant to astrophysical and experimental environments. Here, we analyze the asymptotic behavior of both primary MRI and secondary modes in this regime and provide explanations for the behaviors observed in Figures 3 and 4

\subsection{Asymptotic Behavior of Primary MRI Modes}

In order to better appreciate the asymptotic behavior of the parasitic instabilities, we need to consider the asymptotic behavior of the primary MRI modes. The quantities that define the structure of these modes, as well as some relationships between various timescales and amplitudes, are of particular importance and we briefly summarize them here. We focus our attention in the case of a Keplerian shear profile, i.e., $q=3 / 2$.

In ideal MHD, i.e., $\Lambda_{\nu}, \Lambda_{\eta} \gg 1$, the maximum growth achieved by the MRI is $\Gamma_{\max }=(3 / 4) \Omega_{0}$, and corresponds to the mode with wavenumber $K_{\max }=\sqrt{15 / 16} \Omega_{0} / \bar{v}_{\mathrm{A} z}$. The fastest growth rate is related to the Alfvén frequency, $\omega_{\mathrm{A} z}=K_{\max } \bar{v}_{\mathrm{A} z}$, associated with the background magnetic field $\bar{B}_{z}$, via $\Gamma_{\max }=\sqrt{3 / 5} \omega_{\mathrm{A} z}$ with $\omega_{\mathrm{A} z}=\sqrt{15 / 16} \Omega_{0}$. The magnetic field and velocity field amplitudes for the fastest primary MRI mode are related via $V_{0}=\sqrt{3 / 5} B_{0} / \sqrt{4 \pi \rho}$ (Pessah et al. 2006a). The angles characterizing the planes which contain the velocity and magnetic fields of the fastest growing MRI mode are given by $\sin \theta_{\mathrm{V}}=-\cos \theta_{\mathrm{B}}=1 / \sqrt{2}$.

In the inviscid, resistive MHD limit, i.e., $\Lambda_{\nu} \gg 1$ and $\Lambda_{\eta} \ll 1$, the fastest MRI growth rate $\Gamma_{\max }=$ $(3 / 4) \Lambda_{\eta} \Omega_{0}$, corresponds to the mode with wavenumber $K_{\max }=\sqrt{3 / 4} \Lambda_{\eta}\left(\Omega_{0} / \bar{v}_{\mathrm{A} z}\right)$. In this case, the various relevant inverse timescales are related via $\Gamma_{\max }=\sqrt{3 / 4} \omega_{\mathrm{A} z}=$ $\omega_{\eta}$, where $\omega_{\mathrm{A} z}=K_{\max } \bar{v}_{\mathrm{A} z}=\sqrt{3 / 4} \Lambda_{\eta} \Omega_{0}$ is the Alfvén frequency and $\omega_{\eta}=\eta K_{\max }^{2}=(3 / 4) \Lambda_{\eta} \Omega_{0}$ is the inverse of the resistive timescale across a lengthscale of the order of the MRI wavelength, i.e., $K_{\max }^{-1}$. The magnetic field and velocity field amplitudes for the fastest primary MRI mode are related via $V_{0}=\sqrt{3} \Lambda_{\eta} B_{0} / \sqrt{4 \pi \rho}$ (Pessah \& Chan 2008), and thus 
$V_{0} / B_{0} \rightarrow 0$ in the limit $\Lambda_{\eta} \ll 1$. The planes containing the fastest growing MRI velocity and magnetic fields are characterized by the angles $\sin \theta_{\mathrm{V}}=(5 / 8) \Lambda_{\eta}$ and $\cos \theta_{\mathrm{B}}=-\Lambda_{\eta}$.

\subsection{Asymptotic Behavior of Parasitic Growth Rates}

The dependence of the growth rate of the fastest parasitic modes on the amplitude of the primary MRI magnetic field, $B_{0}$, is shown in Figure 6. The open circles identify Kelvin-Helmholtz modes that feed off the MRI velocity field at $\theta \simeq \theta_{\mathrm{V}}$, which exhibit the fastest growing rates for $\Lambda_{\eta} \geq 1$. The filled circles correspond to tearing modes, that feed off the MRI currents at $\theta \simeq \theta_{\mathrm{B}}$. These are the fastest growing secondary instabilities for $\Lambda_{\eta}<1$. The following equation 3 , shown as dashed lines in Figure 6, provide an excellent description of the fastest growth rates associated with KelvinHelmholtz modes

$$
\begin{array}{ll}
s_{\max }=0.20 B_{0} & \text { for } \quad \theta \simeq \theta_{\mathrm{V}}, \Lambda_{\eta}>1, \\
s_{\max }=0.10 B_{0} & \text { for } \quad \theta \simeq \theta_{\mathrm{V}}, \Lambda_{\eta}=1,
\end{array}
$$

while the growth rates of the tearing-modes is described by

$$
s_{\max }=0.15 B_{0} \Lambda_{\eta} \quad \text { for } \quad \theta \simeq \theta_{\mathrm{B}}, \Lambda_{\eta} \leq 1 .
$$

The critical value of the Elsasser number at which the fastest growing tearing modes (along the direction $\theta \simeq \theta_{\mathrm{B}}$ ) grow faster than the fastest growing Kelvin-Helmholtz modes (along the direction $\theta \simeq \theta_{\mathrm{V}}$ ) is very close to (but slightly less than) unity, i.e., $\Lambda_{\eta}^{\mathrm{c}} \lesssim 1$, see Figure 5] The existence of a an order unity critical Elsasser number that dictates the linear evolution of the MRI has already been appreciated on both numerical (Sano et al. 1998; Fleming et al. 2000) and analytical grounds (see, e.g., Sano \& Miyama 1999; Pessah \& Chan 2008). Here we posit that this critical Elsasser number also distinguishes which type of parasitic instability dominates the subsequent evolution of the MRI. In the remainder of this Section we argue, and demonstrate in Section 6 that there are two regimes such that the fastest growing parasitic modes correspond to

$$
\begin{array}{ll}
\Lambda_{\eta}>\Lambda_{\eta}^{\mathrm{c}} \simeq 1 & \rightarrow \quad \text { Kelvin-Helmholtz } \\
\Lambda_{\eta}<\Lambda_{\eta}^{\mathrm{c}} \simeq 1 & \rightarrow \quad \text { Tearing Modes }
\end{array}
$$

The linear dependence of the fastest parasitic growth rates on the amplitude $B_{0}$, or $V_{0}$, when both viscous and resistive effects are unimportant is to be expected, see Equations 27) and (28). However, the linear dependence of the fastest growth rates on $B_{0}$ and $\Lambda_{\eta}$ for $\Lambda_{\eta} \leq 1$ is not a trivial result. These dependencies are responsible for the asymptotic behaviors described in Section 4.

\subsubsection{Kelvin-Helmholtz Modes}

For the fastest growing Kelvin-Helmholtz instabilities with $\Lambda_{\nu} \gg 1$ and $\Lambda_{\eta}>1$, the ratio of the horizontal parasitic wavenumber to the wavenumber of the fastest growing primary MRI mode depends very weakly on either $\Lambda_{\nu}$ or $\Lambda_{\eta}$, see Table 1, with

$$
\frac{k_{\mathrm{h}, \max }}{K_{\max }} \simeq 0.59 \text { for } \quad \Lambda_{\nu} \gg 1, \Lambda_{\eta}>1
$$

3 Although not shown in Fig. 6 the growth rates of the (sub-dominant) Kelvin-Helmholtz modes are given by $s_{\max }=0.37 B_{0} \Lambda_{\eta}^{2}$ for $\theta \simeq$ $\theta_{\mathrm{V}}, \Lambda_{\eta} \leq 1$. There does not seem to be simple scaling relations for the (subdominant) tearing modes in the regime $\Lambda_{\nu} \gg 1$ and $\Lambda_{\eta}>1$ for $\theta \simeq \theta_{\mathrm{B}}$.

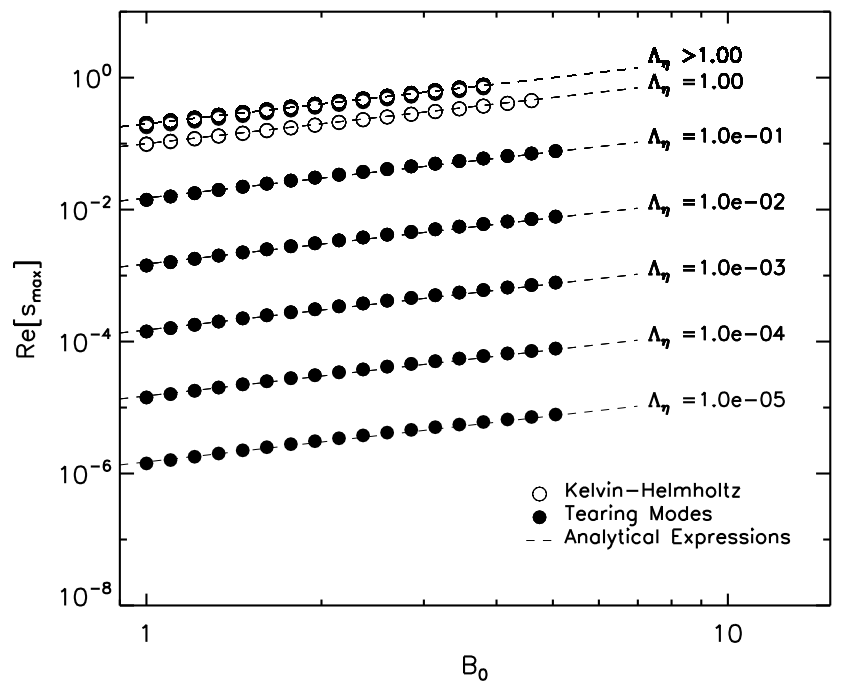

FIG. 6.- Growth rates for the fastest growing parasitic instabilities as a function of the amplitude of the MRI magnetic field $B_{0}$. The values of $\Lambda_{\eta}$ associated with each set of data points is shown on the right. Open circles represent Kelvin-Helmholtz modes, which exhibit the fastest growing rates for $\Lambda_{\eta} \geq 1$. Filled circles represent tearing modes, which exhibit the fastest growing rates for $\Lambda_{\eta}<1$. In all cases, the data points are drawn for $B_{0} \leq B_{0}^{\text {sat }}(\nu, \eta)$. The dashed lines, proportional to $B_{0}$, correspond to the analytical expressions in Equations 38-40.

Therefore, restoring the physical dimensions into Equation (38) we obtain

$$
s_{\max } \simeq 0.44 \frac{\omega_{\mathrm{KH}, 0} \Omega_{0}}{\omega_{\mathrm{A} z}} \simeq 0.45 \omega_{\mathrm{KH}, 0},
$$

where $\omega_{\mathrm{A} z}$ is the ideal Alfvén frequency and $\omega_{\mathrm{KH}, 0} \equiv$ $k_{\mathrm{h}} V_{0}$, which corresponds to the growth rate associated with a Kelvin-Helmholtz instability feeding off a velocity field discontinuity of amplitude $V_{0}$. Therefore, the amplitude of the MRI magnetic field at which the growth rate of the parasites matches the growth of the primary mode can be obtained using (38), which leads, in agreement with Figure 3, to

$$
B_{0}^{\text {sat }} \simeq 3.8 \text { for } \Lambda_{\nu} \gg 1, \Lambda_{\eta}>1 .
$$

\subsubsection{Tearing Modes}

For the fastest growing tearing modes with $\Lambda_{\nu} \gg 1$ and $\Lambda_{\eta}<1$, the ratio of the horizontal parasitic wavenumber to the wavenumber of the fastest growing primary MRI mode depends very weakly on either $\Lambda_{\nu}$ or $\Lambda_{\eta}$, see Table 1 ,

$$
\frac{k_{\mathrm{h}, \max }}{K_{\max }} \simeq 0.48 \text { for } \Lambda_{\nu} \gg 1, \Lambda_{\eta}<1 .
$$

Therefore, Equation (40) leads to

$$
s_{\max } \simeq 0.31 \frac{\omega_{\mathrm{A} 0} \omega_{\mathrm{A} z}}{\omega_{\eta}} \simeq 0.36 \omega_{\mathrm{A} 0},
$$

where we have defined $\omega_{\mathrm{A} 0} \equiv k_{\mathrm{h}} B_{0} / \sqrt{4 \pi \rho}$, as the Alfvén frequency associated with the horizontal MRI magnetic field $B_{0}$ and used that $\omega_{\eta}=\sqrt{3 / 4} \omega_{\mathrm{A} z}$. The amplitude of the magnetic field that the MRI needs to grow to in order for the growth rate of the parasites to be as large as that corresponding to the primary mode is obtained using Equation (40). This leads, in agreement with Figure 3, to

$$
B_{0}^{\text {sat }} \simeq 5.0 \text { for } \Lambda_{\nu} \gg 1, \Lambda_{\eta}<1 .
$$




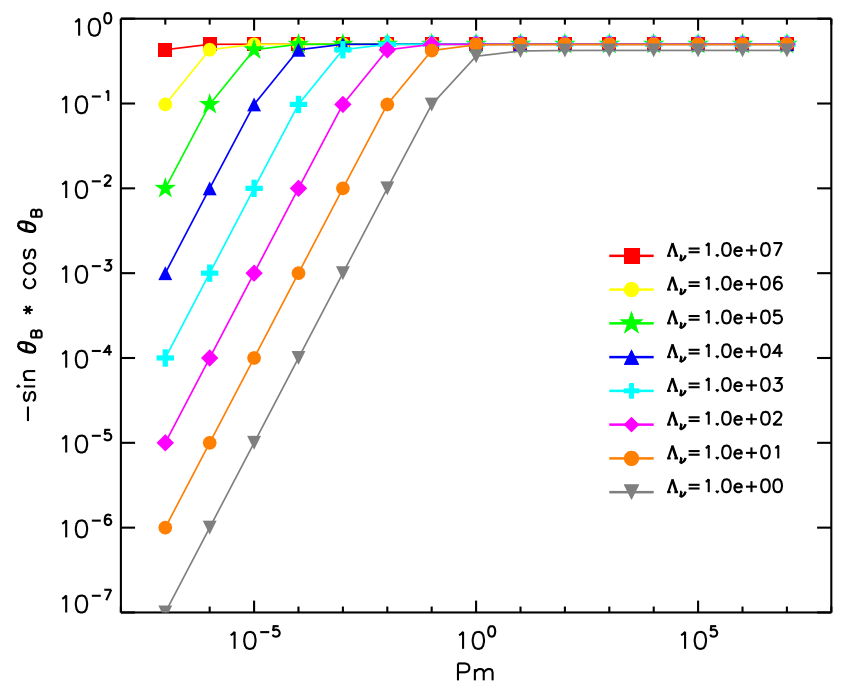

FIG. 7.- Plot of the function $\left|\sin \theta_{\mathrm{B}} \cos \theta_{\mathrm{B}}\right|$, where $\theta_{\mathrm{B}}$ corresponds to the angle subtended by the magnetic field of the fastest MRI mode and the radial direction. In the ideal limit, with $\Lambda_{\eta} \gg \Lambda_{\nu} \gg 1,\left|\sin \theta_{\mathrm{B}} \cos \theta_{\mathrm{B}}\right| \rightarrow$ $1 / \sqrt{2}$, while in the inviscid, resistive limit, i.e., $\Lambda_{\nu} \gg 1$ and $\Lambda_{\eta} \ll 1$, $\left|\sin \theta_{\mathrm{B}} \cos \theta_{\mathrm{B}}\right| \rightarrow \operatorname{Pm} \Lambda_{\nu} \equiv \Lambda_{\eta}$. Because $B_{0}^{\text {sat }} \rightarrow$ const., the behavior of this function is responsible for the decrease of the MRI stress shown in Figure 4

We can formulate a heuristic argument for the existence of an asymptotic limit for the amplitude $B_{0}$ in this regime. The analysis of the classical tearing mode instability, where $B_{0}=B_{0} \tanh (K z)$, with $K$ fixed, leads to growth rates $\gamma \sim\left(\eta K^{2}\right)^{\alpha}\left(K B_{0}\right)^{1-\alpha}$ with $\alpha=1 / 2$ or $\alpha=3 / 5$ depending on the details of how the problem is solved in the resistive layer (see, e.g., Furth et al. 1963; Sturrock 1994). For $\eta \gg 1 \gg \nu$, there are only two characteristic scales in the problem, the resistive timescale $\left(\eta K^{2}\right)^{-1}$ and the Alfvén timescale $\left(K B_{0}\right)^{-1}$. Therefore, the growth rate of the parasites must be $s \sim\left(\eta K^{2}\right)^{\alpha}\left(K B_{0}\right)^{1-\alpha}=\eta^{\alpha} K^{1+\alpha} B_{0}^{1-\alpha}$, with $0 \leq \alpha \leq 1$. At a fixed scale $K$, the growth rate of the secondaries is proportional to a positive power of the resistivity, $s \sim \eta^{\alpha}$. However, if the scale $K$ is given by the fastest growing MRI mode then $K \sim \eta^{-1}$ and thus $s_{\max } \sim\left(B_{0}\right)^{1-\alpha} / \eta$. In this case, because $\Gamma_{\max } \sim \eta^{-1}$, the amplitude $B_{0}$ at which $s_{\max }=\Gamma_{\max }$ must be independent of $\eta$, i.e.,

$$
s_{\max } \simeq \Gamma_{\max } \sim\left(\eta \frac{1}{\eta^{2}}\right)^{\alpha}\left(\frac{B_{0}}{\eta}\right)^{1-\alpha} \sim \frac{1}{\eta} .
$$

Therefore, $B_{0} \rightarrow$ const. for $\Lambda_{\eta} \ll 1$, i.e., the amplitude to which the primary MRI magnetic field needs to grow to in order for both growth rates to become comparable is independent of the Elsasser number. This means that, as resistivity increases the magnetic field that can be generated by the MRI before the tearing modes become dynamically important reaches an asymptotic, constant value. However, as we showed in Section 4 the associated stress decreases with $\operatorname{Pm} \Lambda_{\nu} \equiv \Lambda_{\eta}$ for $\Lambda_{\eta} \ll 1$. This is because, in this limit, $V_{0} / B_{0} \ll 1$ and the stress behaves like $\bar{T}_{r \phi} \sim B_{0}^{2}\left|\sin \theta_{\mathrm{B}} \cos \theta_{\mathrm{B}}\right| \sim \operatorname{Pm} \Lambda_{\nu} \equiv \Lambda_{\eta}$, see Figure 7.

\section{KELVIN-HELMHOLTZ AND TEARING EIGENMODES}

Throughout this paper we have stated that the fastest parasites are related to Kelvin-Helmholtz and tearing mode instabilities in the regimes $\Lambda_{\eta} \gtrsim 1$ and $\Lambda_{\eta}<1$, respectively. The reason to delay presenting the rigorous evidence support- ing these claims up to this point is based on the existence of the asymptotic regimes and scaling laws presented in Section 5. We can now focus on the region of parameter space with "moderate" Elsasser number, $\Lambda_{\eta} \simeq 1$, where changes in $\Lambda_{\eta}$ produce non-trivial modifications to the parasitic mode structure. With this knowledge, and the insight gained in Section 5, it is straightforward to describe the structure of these modes in the limits of large and small Elsasser numbers.

\subsection{Physical Structure of Parasitic Modes}

In order to understand the nature of the most relevant secondary modes we analyze the structure of their velocity $\delta \boldsymbol{v}_{\|}$ and magnetic fields $\delta \boldsymbol{B}_{\|}$along the directions associated with their fastest growth, i.e., $\theta=\theta_{\max }$. For fixed values of the dissipation coefficients, the growth rates of the secondary instabilities peak around directions which are almost aligned with either the velocity or magnetic fields of the primary MRI mode, i.e., $\theta_{\max } \simeq \theta_{\mathrm{V}}$ for $\Lambda_{\eta} \gg 1$ and $\theta_{\max } \simeq \theta_{\mathrm{B}}$ for $\Lambda_{\eta} \ll 1$, see Figure 5. Motivated by the physical characteristics of the Kelvin-Helmholtz and tearing modes, we calculate the vorticity $\delta \boldsymbol{\omega}_{\perp}$ and current density $\delta \boldsymbol{j}_{\perp}$ associated with the parasitic modes in the directions that are perpendicular to the planes defined by $\theta \equiv \theta_{\max }$. We also analyze the Lagrangian displacements $\xi_{z}$ induced by the secondary modes which provide useful complementary information regarding the reconnection of the magnetic field associated with the MRI.

Understanding the structure of the secondary modes requires calculating $\delta \boldsymbol{v}(x, y, z)$ and $\delta \boldsymbol{b}(x, y, z)$. However, in the incompressible limit we can calculate $\delta \boldsymbol{v}_{\|}, \delta \boldsymbol{B}_{\|}, \delta \boldsymbol{\omega}_{\perp}$, and $\delta \boldsymbol{j}_{\perp}$ directly in terms of the Fourier coefficients for $\delta v_{z}$ and $\delta b_{z}$ obtained from Equations (27) and (28). In order to do this, it is useful to define a new coordinate system $(h, p, z)$ that is rotated with respect to $(x, y, z)$ about the $\check{z}$ direction by $\theta_{\max }$. We define the coordinates $h$ and $p$ such that they increase along the direction of the versors $\check{\boldsymbol{k}}_{\mathrm{h}}$ and $\check{\boldsymbol{k}}_{\mathrm{p}}$, respectively, with $\check{\boldsymbol{k}}_{\mathrm{h}} \times \check{\boldsymbol{k}}_{\mathrm{p}}=\check{\boldsymbol{z}}$ and $\check{\boldsymbol{k}}_{\mathrm{p}} \times \check{\boldsymbol{z}}=\check{\boldsymbol{k}}_{\mathrm{h}}$, see Figure 2 .

\subsubsection{Velocity and Magnetic Fields}

In the new coordinate system, the velocity and magnetic fields of the parasitic modes have components

$$
\begin{aligned}
\delta \boldsymbol{v} & =\delta v_{h} \check{\boldsymbol{k}}_{\mathrm{h}}+\delta v_{p} \check{\boldsymbol{k}}_{\mathrm{p}}+\delta v_{z} \check{\boldsymbol{z}}, \\
\delta \boldsymbol{B} & =\delta B_{h} \check{\boldsymbol{k}}_{\mathrm{h}}+\delta B_{p} \check{\boldsymbol{k}}_{\mathrm{p}}+\delta B_{z} \check{\boldsymbol{z}} .
\end{aligned}
$$

Since $\boldsymbol{k}_{\mathrm{h}} \cdot \boldsymbol{x} \equiv k_{\mathrm{h}} h$, the secondary perturbations $\delta v_{z}$ and $\delta B_{z}$ in Equations (23) and (24) become

$$
\begin{aligned}
\delta v_{z}(h, z) & =\sum_{n=-\infty}^{\infty} \alpha_{n} e^{-i\left(n+k_{z}\right) z} e^{-i k_{\mathrm{h}} h}, \\
\delta B_{z}(h, z) & =\sum_{n=-\infty}^{\infty} \beta_{n} e^{-i\left(n+k_{z}\right) z} e^{-i k_{\mathrm{h}} h} .
\end{aligned}
$$

Because we are considering an incompressible fluid, $\delta \boldsymbol{v} \cdot \check{\boldsymbol{k}}_{\mathrm{h}}=$ $\partial_{z} \delta v_{z} /\left(i k_{\mathrm{h}}\right)$ and $\delta \boldsymbol{B} \cdot \check{\boldsymbol{k}}_{\mathrm{h}}=\partial_{z} \delta B_{z} /\left(i k_{\mathrm{h}}\right)$. We can thus calculate the projection of the velocity and magnetic field of a given parasitic mode onto the plane defined by $\left(\check{\boldsymbol{k}}_{\mathrm{h}}, \check{\boldsymbol{z}}\right)$, i.e.,

$$
\begin{aligned}
\delta \boldsymbol{v}_{\|} & =\left(\delta \boldsymbol{v} \cdot \check{\boldsymbol{k}}_{\mathrm{h}}\right) \check{\boldsymbol{k}}_{\mathrm{h}}+\delta v_{z} \check{\boldsymbol{z}}, \\
\delta \boldsymbol{B}_{\|} & =\left(\delta \boldsymbol{B} \cdot \check{\boldsymbol{k}}_{\mathrm{h}}\right) \check{\boldsymbol{k}}_{\mathrm{h}}+\delta B_{z} \check{\boldsymbol{z}},
\end{aligned}
$$

\footnotetext{
${ }^{4}$ In order to simplify notation, in this section we refer to the versor associated with the direction of fastest growth, i.e. $\check{\boldsymbol{k}}_{\mathrm{h}, \max }$, simply as $\breve{\boldsymbol{k}}_{\mathrm{h}}$
} 

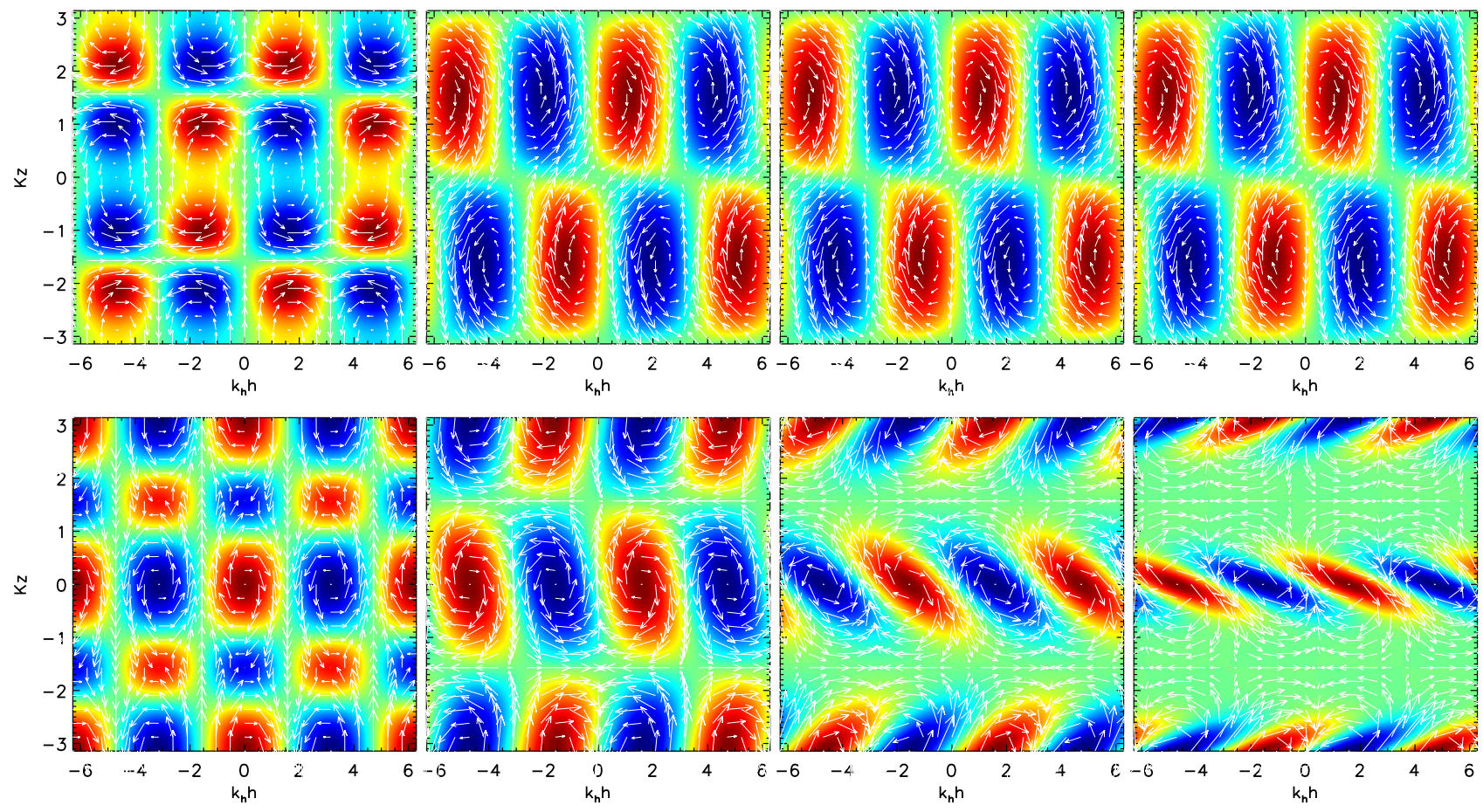

FIG. 8. - Physical structure of the fastest parasitic modes for $\Lambda_{\eta}=\left\{0.1,1,10,10^{2}\right\}$, from left to right, with $\Lambda_{\nu} \gg 1$. The arrows in the upper and lower panels correspond to the projections of the velocity, $\delta \boldsymbol{v}_{\|}(h, z)$, and magnetic field, $\delta \boldsymbol{B}_{\|}(h, z)$, of the parasitic modes onto the plane defined by the $z$-axis and the direction $\theta_{\max }$. The color contours correspond to the associated vorticity, $\delta \boldsymbol{\omega}_{\perp}$, and current density, $\delta \boldsymbol{j}_{\perp}$, projected in the direction perpendicular to $\theta_{\text {max }}$, see Figs. 1 2 and 5 The left-most (upper and lower) panels, show the vorticity and current density patterns characteristic of the tearing mode instability, (see Fig. 5.7 in Boyd \& Sanderson 2003). The next set of three upper panels show clear signatures of the Kelvin-Helmholtz instability in the vorticity contours (see Fig. 1.7.2 in Batchelor 2000). The lower panels show the (less familiar) current density perturbations associated with these Kelvin-Helmholtz modes.

just in terms of the Fourier coefficients $\left\{\alpha_{n}\right\}$ and $\left\{\beta_{n}\right\}$ as

$$
\begin{aligned}
\delta \boldsymbol{v}_{\|}(h, z) & =\frac{1}{k_{\mathrm{h}}} \sum_{n=-\infty}^{\infty}\left(n+k_{z}\right) \alpha_{n} e^{-i\left(n+k_{z}\right) z} e^{-i k_{\mathrm{h}} h} \check{\boldsymbol{k}}_{\mathrm{h}} \\
& +\sum_{n=-\infty}^{\infty} \alpha_{n} e^{-i\left(n+k_{z}\right) z} e^{-i k_{\mathrm{h}} h} \check{\boldsymbol{z}} \\
\delta \boldsymbol{B}_{\|}(h, z) & =\frac{1}{k_{\mathrm{h}}} \sum_{n=-\infty}^{\infty}\left(n+k_{z}\right) \beta_{n} e^{-i\left(n+k_{z}\right) z} e^{-i k_{\mathrm{h}} h} \check{\boldsymbol{k}}_{\mathrm{h}} \\
& +\sum_{n=-\infty}^{\infty} \beta_{n} e^{-i\left(n+k_{z}\right) z} e^{-i k_{\mathrm{h}} h} \check{\boldsymbol{z}}
\end{aligned}
$$

Note that the components of the three-dimensional velocity and magnetic fields that are orthogonal to these planes are independent of the coordinate $p$. Thus, using the fact that the divergence is invariant under rotations, we conclude that the divergence of the two-dimensional vector fields that lie on the plane $\left(\check{\boldsymbol{k}}_{\mathrm{h}}, \check{\boldsymbol{z}}\right)$ should vanish, i.e., $\boldsymbol{\nabla} \cdot \delta \boldsymbol{v}_{\|}=\boldsymbol{\nabla} \cdot \delta \boldsymbol{B}_{\|}=0$.

\subsubsection{Vorticity and Current Density}

The components of the vorticity and current density perpendicular to the plane defined by $\left(\check{\boldsymbol{k}}_{\mathrm{h}}, \check{\boldsymbol{z}}\right)$, i.e., $\delta \boldsymbol{\omega}_{\perp}=\delta \omega_{\perp} \check{\boldsymbol{k}}_{\mathrm{p}}$ and $\delta \boldsymbol{j}_{\perp}=\delta j_{\perp} \check{\boldsymbol{k}}_{\mathrm{p}}$, are given by

$$
\begin{aligned}
\delta \omega_{\perp} & =\delta \boldsymbol{\omega} \cdot \check{\boldsymbol{k}}_{\mathrm{p}}=(\boldsymbol{\nabla} \times \delta \boldsymbol{v}) \cdot \check{\boldsymbol{k}}_{\mathrm{p}}=\left(\boldsymbol{\nabla} \times \delta \boldsymbol{v}_{\|}\right) \cdot \check{\boldsymbol{k}}_{\mathrm{p}}, \\
\delta j_{\perp} & =\delta \boldsymbol{j} \cdot \check{\boldsymbol{k}}_{\mathrm{p}}=(\boldsymbol{\nabla} \times \delta \boldsymbol{B}) \cdot \check{\boldsymbol{k}}_{\mathrm{p}}=\left(\boldsymbol{\nabla} \times \delta \boldsymbol{B}_{\|}\right) \cdot \check{\boldsymbol{k}}_{\mathrm{p}},
\end{aligned}
$$

where the action of the curl operators is given by

$$
\begin{aligned}
& \boldsymbol{\nabla} \times \delta \boldsymbol{v}_{\|}=\left(\partial_{z} \delta v_{h}-\partial_{h} \delta v_{z}\right) \check{\boldsymbol{k}}_{\mathrm{p}}, \\
& \boldsymbol{\nabla} \times \delta \boldsymbol{j}_{\|}=\left(\partial_{z} \delta B_{h}-\partial_{h} \delta B_{z}\right) \check{\boldsymbol{k}}_{\mathrm{p}} .
\end{aligned}
$$

Therefore, using Equations (56) and (57), we obtain

$$
\begin{gathered}
\delta \boldsymbol{\omega}_{\perp}=\frac{i \check{\boldsymbol{k}}_{\mathrm{p}}}{k_{\mathrm{h}}} \sum_{n=-\infty}^{\infty}\left[k_{\mathrm{h}}^{2}+\left(n+k_{z}\right)^{2}\right] \alpha_{n} e^{-i\left(n+k_{z}\right) z} e^{-i k_{\mathrm{h}} h} \\
\delta \boldsymbol{j}_{\perp}=\frac{i \check{\boldsymbol{k}}_{\mathrm{p}}}{k_{\mathrm{h}}} \sum_{n=-\infty}^{\infty}\left[k_{\mathrm{h}}^{2}+\left(n+k_{z}\right)^{2}\right] \beta_{n} e^{-i\left(n+k_{z}\right) z} e^{-i k_{\mathrm{h}} h} .
\end{gathered}
$$

\subsubsection{Lagrangian Displacement}

The vertical Lagrangian displacement $\xi_{z}$ provides useful complementary information to help us identify the physical nature of the eigenmodes. The rate of change in the Lagrangian displacement $\boldsymbol{\xi}$ with respect to a point moving with velocity $\boldsymbol{v}$ is given by

$$
\frac{d \boldsymbol{\xi}}{d t} \equiv \frac{\partial \boldsymbol{\xi}}{\partial t}+(\boldsymbol{v} \cdot \boldsymbol{\nabla}) \boldsymbol{\xi}=\delta \boldsymbol{v}+\boldsymbol{\xi} \cdot \boldsymbol{\nabla} \boldsymbol{v} .
$$

In the periodic background provided by the primary MRI mode, the Lagrangian displacement is of the form $\boldsymbol{\xi}(\boldsymbol{x}, t)=$ $\boldsymbol{\xi}_{0}(z) \exp [s t-i \boldsymbol{k} \cdot \boldsymbol{x}]$ with $\boldsymbol{\xi}_{0}(\boldsymbol{x}+2 \pi / K \check{\boldsymbol{z}})=\boldsymbol{\xi}_{0}(\boldsymbol{x})$. Therefore, considering the MRI velocity field, $\Delta \boldsymbol{v}=\boldsymbol{V}_{0} \sin (K z)$, as the background velocity, we obtain $\xi_{z}$ in terms of the verti- 


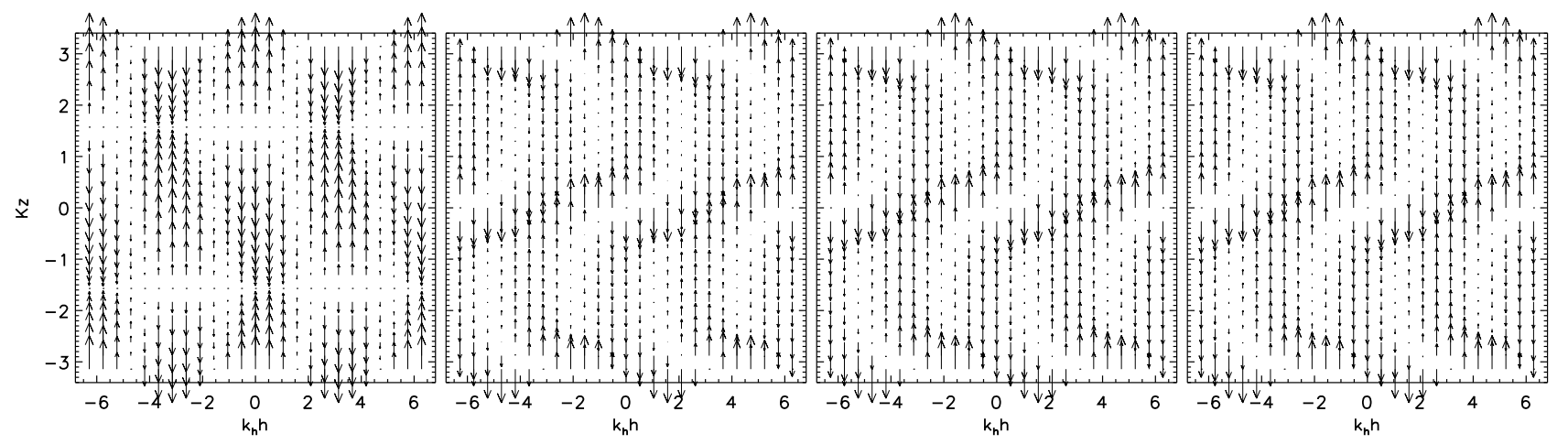

FIG. 9.- Vertical Lagrangian displacement $\xi_{z}(h, z)$ corresponding to the fastest growing parasitic modes projected onto the plane defined by the $z$-axis and the direction $\theta_{\max }$. The panels correspond to $\Lambda_{\eta}=\left\{0.1,1,10,10^{2}\right\}$, from left to right, with $\Lambda_{\nu} \gg 1$. The convergence of the displacement field toward the null surfaces of the MRI magnetic field, $\Delta B \propto \cos (K z)$, located at $K z= \pm n \pi / 2$, is evident in the leftmost panel. This feature is characteristic of a tearing mode; the associated motions are ultimately responsible for reconnecting the MRI magnetic field and limiting its growth. The next three panels show the displacements associated with the fastest Kelvin-Helmholtz modes, which mainly tend to bend the MRI magnetic field without directly promoting reconnection.

cal Eulerian velocity $\delta v_{z}$ in Equation (52) as

$$
\xi_{z}(h, z)=\frac{\delta v_{z}(h, z)}{s-i \boldsymbol{k}_{\mathrm{h}} \cdot \Delta \boldsymbol{v}} .
$$

\subsection{Parasitic Mode Identification}

In Section 5 we showed that the eigenvalues corresponding to the growth rates of the parasitic modes reached well defined asymptotic regimes for $\Lambda_{\eta} \ll 1$ and $\Lambda_{\eta} \gg 1$ in the limit $\Lambda_{\nu} \gg 1$. This must also be true for the eigenmodes. It is then only necessary to explore in detail the behavior of the modes for moderate values of $\Lambda_{\eta}$. We thus focus our attention in the region of parameter space spanned by $\Lambda_{\eta}=\left\{0.1,1,10,10^{2}\right\}$. The structures of the fastest parasitic modes are shown in Figure 8 , from left to tight. The projections of the velocity and magnetic fields onto the planes defined by $\left(\check{\boldsymbol{k}}_{\mathrm{h}}, \check{\boldsymbol{z}}\right)$, i.e., $\delta \boldsymbol{v}_{\|}(h, z)$ and $\delta \boldsymbol{B}_{\|}(h, z)$, are shown with white arrows in the upper and lower panels, respectively. The color contours show the projection of the vorticity and current density along the direction $\breve{\boldsymbol{k}}_{\mathrm{p}}$ (perpendicular to the page), i.e., $\delta \boldsymbol{\omega}_{\perp}$ and $\delta \boldsymbol{j}_{\perp}$. The red and blue colors correspond to the maximum positive and minimum negative values associated with the vorticity and current density of each mode.

\subsubsection{Tearing Modes}

For the Elsasser number $\Lambda_{\eta}=0.1$, the versor characterizing the direction of fastest growth, $\check{\boldsymbol{k}}_{\mathrm{h}}$, points in the direction $\theta_{\max } \simeq \theta_{\mathrm{B}}$, see Fig. (5) and Table1 This mode, shown in the leftmost (upper and lower) panels of Figure 8 , feeds off the current density of the primary MRI mode. The corresponding mode structure resembles closely the perturbations in the current density and induced vorticity patterns expected in the analysis of the stability of a set of equidistant current sheets distributed along the $\check{z}$ direction and alternating sense according to $\pm \check{\boldsymbol{k}}_{\mathrm{p}}$, (see, Figure 5.7 in Boyd \& Sanderson 2003).

The current density of the secondary modes presents maxima and minima along the planes $K z= \pm n \pi / 2$ where the magnetic field of the primary mode, $\Delta \boldsymbol{B}=\boldsymbol{B}_{0} \cos (K z)$, reverses sign. Thus, the fluctuations induced by these fastest resistive secondary modes tend to promote reconnection of the MRI field. This can be better appreciated by analyzing the vertical Lagrangian displacement shown in the leftmost panel of Figure 9. There is another set of periodic maxima and minima in the current density fluctuations that lie on planes $K z=0, \pm n \pi$; these are the locations where the currents associated with the MRI magnetic field vanish. Thus, these current density perturbations do not seem to be due to the unstable configuration presented by the MRI currents themselves. They rather seem to be needed to satisfy the periodic constraints on the scale of the unstable MRI mode.

The observed mode structure is qualitatively insensitive to the value of the Elsasser number as long as $\Lambda_{\eta}<1$ and $\Lambda_{\nu} \gg 1$, the growth rates and lengthscales associated with each value of $\Lambda_{\eta}$ change, of course, as discussed in Section 5 We thus conclude that the fastest parasitic modes correspond to tearing modes for $\Lambda_{\eta}<1$. These parasitic modes are enabled by non-zero resistivity and are thus absent in the ideal MHD regime studied by Goodman \& Xu (1994).

\subsubsection{Kelvin-Helmholtz Modes}

For the Elsasser numbers $\Lambda_{\eta}=\left\{1,10,10^{2}\right\}$, the versors $\check{\boldsymbol{k}}_{\mathrm{h}}$ characterizing the direction of fastest growth point in the direction $\theta_{\max } \simeq \theta_{\mathrm{V}}$ for the three rightmost sets of panels in Fig. (5), see also Table 1. These modes, shown in the three rightmost (upper and lower) panels of Figure 8 , feed off the shear in the velocity field of the corresponding primary MRI modes. The velocity and vorticity fields show a periodic structure similar to what is expected from the stability analysis of a periodic set of equidistant vortex sheets distributed along the $\check{\boldsymbol{z}}$ direction and alternating sense according to $\pm \check{\boldsymbol{k}}_{\mathrm{p}}$.

The mode structure in the velocity and vorticity fields, as well as the growth rates and lengthscales, associated with each value of $\Lambda_{\eta}$ are quantitatively insensitive to the value of the Elsasser number as long as $\Lambda_{\eta} \geq 1$ and $\Lambda_{\nu} \gg 1$, as discussed in Section 5. The structure of the current densities associated with these modes show some evolution as a function of $\Lambda_{\eta}>1$. However, these modes are not intrinsically modified from pure Kelvin-Helmholtz modes since the amplitude of the fluctuations in the magnetic and current density fields is much smaller than the fluctuations in the velocity and vorticity fields. We thus conclude that the fastest parasitic modes correspond to Kelvin-Helmholtz modes for $\Lambda_{\eta}>1$. In the limit $\Lambda_{\eta} \gg 1$, these correspond of course to the Kelvin-Helmholtz modes alluded to in Goodman \& Xu (1994).

The current density of the secondary modes vanishes along, and also in the vicinity of, the planes $z= \pm n \pi / 2$ where the magnetic field of the primary mode, $\Delta \boldsymbol{B}=\boldsymbol{B}_{0} \cos (K z)$, changes sign. The corresponding Lagrangian displacement associated with these modes for $\Lambda_{\eta}=\left\{1,10,10^{2}\right\}$ are shown in the three rightmost panels in Figure 9, respectively. The fluctuations induced by the fastest growing Kelvin-Helmholtz 

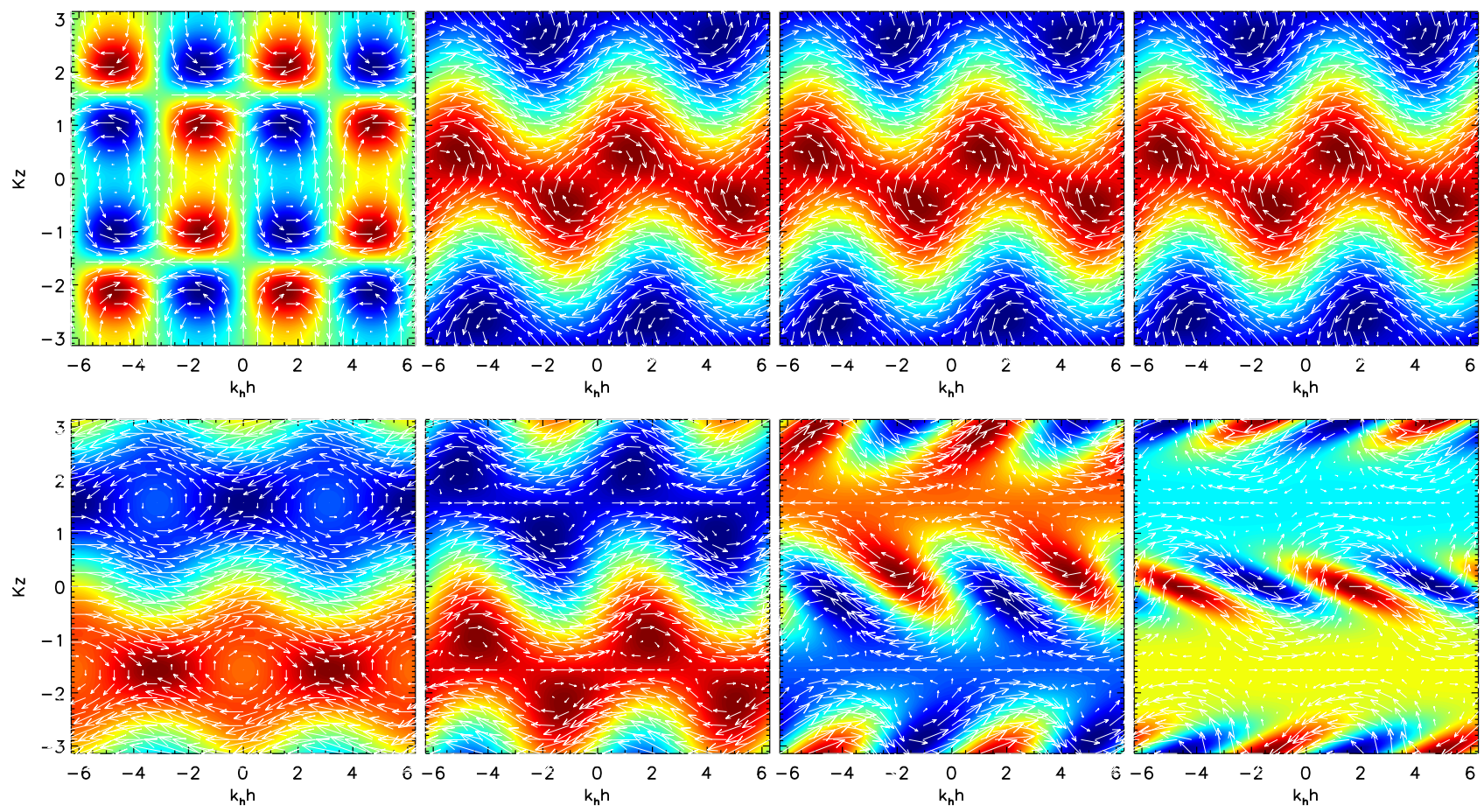

FIG. 10.- Physical structure of the fastest parasitic modes, including the velocity and magnetic fields of the primary MRI modes, for $\Lambda_{\eta}=\left\{0.1,1,10,10^{2}\right\}$, from left to right, with $\Lambda_{\nu} \gg 1$. The arrows in the upper and lower panels correspond, respectively, to the projections of the total (primary plus secondary) velocity, $\Delta \boldsymbol{V}_{\|}+\delta \boldsymbol{v}_{\|}(h, z)$, and magnetic fields, $\Delta \boldsymbol{B}_{\|}+\delta \boldsymbol{B}_{\|}(h, z)$, onto the plane defined by the $z$-axis and the direction $\theta_{\max }$. The color contours correspond to the associated total vorticity and total current density projected onto the direction perpendicular to $\theta_{\max }$.

secondary instabilities tend to bend the horizontal MRI magnetic field without directly promoting their reconnection.

\subsubsection{Inclusion of MRI-background Fields}

The white arrows in the upper and lower panels of Figure 10 show the structure of the velocity and magnetic fields in Figure 8 when the background MRI fields are added to the secondary modes. The projections of the total velocity and magnetic fields onto the planes defined by $\left(\check{\boldsymbol{k}}_{\mathrm{h}}, \check{\boldsymbol{z}}\right)$, are given by $\Delta \boldsymbol{V}_{\|}+\delta \boldsymbol{v}_{\|}(h, z)$ and $\Delta \boldsymbol{B}_{\|}+\delta \boldsymbol{B}_{\|}(h, z)$, where

$$
\begin{aligned}
\Delta \boldsymbol{V}_{\|} & =\boldsymbol{V}_{0} \sin (K z) \cos \left(\theta_{\max }-\theta_{\mathrm{V}}\right), \\
\Delta \boldsymbol{B}_{\|} & =\boldsymbol{B}_{0} \cos (K z) \cos \left(\theta_{\max }-\theta_{\mathrm{B}}\right) .
\end{aligned}
$$

The color contours show the projection of the total vorticity and current density along the direction $\check{\boldsymbol{k}}_{\mathrm{p}}$ (perpendicular to the page), i.e., $\delta \boldsymbol{\omega}_{\perp, 0}+\delta \boldsymbol{\omega}_{\perp}$ and $\delta \boldsymbol{j}_{\perp, 0}+\delta \boldsymbol{j}_{\perp}$. The contributions of the primary MRI mode are given by

$$
\begin{aligned}
\delta \boldsymbol{\omega}_{0, \perp} & =\boldsymbol{\nabla} \times \Delta \boldsymbol{V}_{\|}=V_{0} \cos (K z) \cos \left(\theta-\theta_{\mathrm{V}}\right) \check{\boldsymbol{k}}_{\mathrm{p}} \\
\delta \boldsymbol{j}_{0, \perp} & =\boldsymbol{\nabla} \times \Delta \boldsymbol{B}_{\|}=-B_{0} \sin (K z) \cos \left(\theta-\theta_{\mathrm{B}}\right) \check{\boldsymbol{k}}_{\mathrm{p}}
\end{aligned}
$$

In all cases, the amplitude of the primary mode $V_{0}$ and $B_{0}$ is such that the fastest secondary modes grow as fast as the primary MRI mode, i.e., $s_{\max }\left(\nu, \eta, K_{\max }\right)=\Gamma_{\max }(\nu, \eta)$.

The addition of the MRI fields facilitates the identification of the velocity and magnetic fields that result from the influence of the secondary instabilities with the more familiar structures that are expected from Kelvin-Helmholtz and tearing mode instabilities in periodic backgrounds. In particular, it highlights the presence of " $O$ " and " $X$ " points in the case of the tearing mode and the wave-like structure of the velocity field in the cases associated with Kelvin-Helmholtz modes.

\section{DISCUSSION}

\subsection{This Work in Context}

Understanding from first principles the saturation of MHD turbulence, and the associated angular momentum transport, driven by the MRI in astrophysical disks surrounding young stars and compact objects is a challenging endeavor. The large dynamical range in temperatures, densities, and magnetic fields in these accretion disks implies that the dimensionless variables parameterizing dissipation span a wide range of values. Numerical simulations are indispensable tools for understanding the properties of MRI-driven turbulence in different environments. However, most numerical studies to date have been carried out either without physical dissipation or with dissipation coefficients that have a small overlap with the regions of parameter space relevant to either astrophysical or experimental setups, see Section 4.1. In order to gain physical insight into the regimes of interest, it seems necessary to combine the results of numerical simulations with analytical efforts aim at identifying the essential processes at work.

The idea that secondary instabilities can limit the growth of the MRI, and (more speculatively) play a role in the subsequent turbulent state, was put forward by Goodman \& Xu (1994) and has been considered in more detail by a number of works more recently (Sano 2007; Bodo et al. 2008; Obergaulinger et al. 2009; Latter et al. 2009; Pessah \& Goodman 2009; c.f., Knobloch \& Julien 2005; Jamroz et al. 2008a b). In this paper, we carried out a thorough study of the spectrum and physical structure of parasitic modes that feed off exact MRI modes when the amplitude of the magnetic field produced by the MRI is such that the instantaneous growth rate of the fastest parasitic mode matches that of the fastest primary mode. Following Pessah \& Goodman (2009), we argued that this "saturation" 
amplitude provides an estimate of the magnetic field that can be generated by the MRI before the secondary instabilities suppress its growth significantly. While we invoked several assumptions and approximations in order to make the problem tractable, our approach enabled us to explore dissipative regimes that are relevant to astrophysical and laboratory conditions that lie beyond the regime accessible to current numerical simulations.

We mention two limitations imposed by our assumptions that are worth emphasizing due to their potential significance (see also the related discussion in Section 2.4 in Latter et al. 2009): (i) We neglected the effects of shear on the dynamics of the secondary modes. Non-axisymmetric parasitic modes will shear linearly in time (Goodman \& Xu 1994); therefore, assuming fixed horizontal versors for the parasites is an approximation. (ii) We ignored the explicit coupling between the evolution of the MRI-modes and the secondary instabilities. As the secondary modes grow they drain energy from the primary modes; therefore their growth rates, which rely on the amplitude of the MRI, could be affected. This approximation might also affect the estimates of the saturation amplitude of the MRI modes, since they provide the source of energy that feeds the parasites. Thus the extrapolation of the results presented here to the non-linear regime should be complemented with the pertinent quota of skepticism. Having said this, at present, the properties of parasitic modes described here provide valuable analytical guidance and a basic framework to design and interpret tailored numerical experiments in order to shed light into the non-linear saturation of the MRI.

We summarize here our results and explain how several features of numerical simulations designed to address the saturation of the MRI in protoplanetary disks and accretion disks surrounding compact objects can be interpreted in terms of our findings.

\subsection{Results, Scaling Laws, and Parasitic Mode Identification}

When the magnetic fields involved are weak enough so that the incompressible limit holds, the dynamics of the MRI and the parasitic instabilities depend only on any two independent dimensionless numbers that can be formed using the Elsasser number, $\Lambda_{\eta} \equiv \bar{v}_{\mathrm{A} z}^{2} /\left(\eta \Omega_{0}\right)$, and is its viscous counterpart, $\Lambda_{\nu} \equiv \bar{v}_{\mathrm{A} z}^{2} /\left(\nu \Omega_{0}\right)$. Motivated by recent works that suggest that the saturation amplitude of the MRI depends on the magnetic Prandtl number (Umurhan et al.2007a; Lesur \& Longaretti 2007), we considered $\Lambda_{\nu}$ and Pm $\equiv$ $\Lambda_{\eta} / \Lambda_{\nu}$ as independent parameters.

We found, however, that the parameter driving the behavior of the growth rates of the parasites and the MRI, and thus the magnetic energy density and stresses at saturation, is the Elsasser number $\Lambda_{\eta}$. In particular, we found that, as long as viscous dissipation is small, i.e., $\Lambda_{\nu} \gtrsim 10$, then there exists two regimes (see Figure 11): (i) quasi-ideal MHD, where the physical properties of the MRI and parasitic instabilities are insensitive to dissipation. This holds as long as $\Lambda_{\eta}>1$, which is applicable to the fully ionized regions of accretion disks around compact objects. (ii) inviscid, resistive MHD, where all the relevant dependencies on $\Lambda_{\nu}$ and Pm are only through the product $\operatorname{Pm} \Lambda_{\nu}$, i.e., the Elsasser number $\Lambda_{\eta}$. This regime corresponds to $\Lambda_{\eta}<1$, and characterizes poorly ionized regions of protoplanetary disks (Jin 1996; Sano \& Miyama 1999). The Elsasser number for current Taylor-Couette MRI experiments is close to unity (Nornberg et al. 2010). In this regime, both types of modes present similar growth rates.

We estimated the amplitude of the magnetic fields and

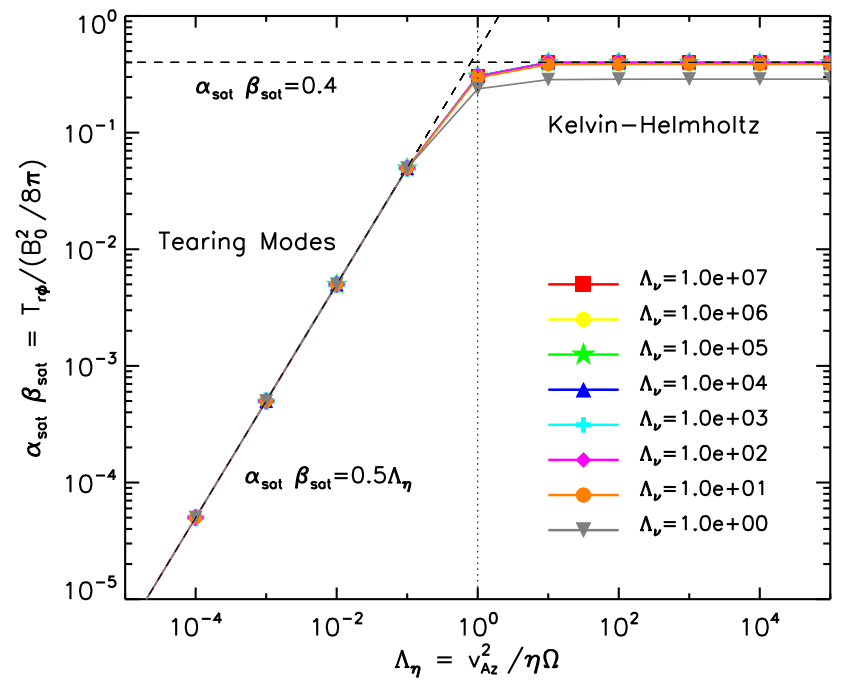

FIG. 11.- Predicted values for the ratio of stress to magnetic energy density, i.e., the product $\alpha_{\mathrm{sat}} \beta_{\mathrm{sat}}$, if saturation occurs when the fastest parasitic and primary MRI growth rates match. For $\Lambda_{\nu} \gtrsim 10$, Equations 70 , and 71 describe the results of the parasitic mode analysis remarkably well. In the quasi-ideal MHD limit, applicable to the fully ionized regions of accretion disks, $\alpha_{\text {sat }} \beta_{\text {sat }}=0.4$. In the inviscid, resistive limit, applicable to poorly ionized regions of protoplanetary disks $\alpha_{\mathrm{sat}} \beta_{\mathrm{sat}}=0.5 \Lambda_{\eta}$. These results are consistent with the numerical simulations shown in Figure 20 of Sano \& Stone (2002), see the discussion in the text for more details. The modes responsible for saturation correspond to Kelvin-Helmholtz and tearing modes for Elsasser numbers $\Lambda_{\eta}$ larger and smaller than unity, respectively. The Elsasser number for current MRI experiments is close to unity. In this regime, both types of modes present similar growth rates.

stresses generated by the MRI when the secondary instabilities become dynamically important. The saturation amplitude of the magnetic fields is fairly insensitive to dissipation with $B_{0}^{\text {sat }} / \bar{B}_{z} \simeq 4-5$. However, the stress behaves very differently. For $\Lambda_{\eta}>1$ the stress reaches and asymptotic value independent of the dissipation coefficients, while for $\Lambda_{\eta}<1$ the stress decreases linearly with decreasing Elsasser number, i.e., $\bar{T}_{r \phi}^{\text {sat }} \propto \Lambda_{\eta}$. This result is consistent with the numerical simulations of resistive MHD shearing boxes carried out by Fleming et al. (2000) and Sano \& Stone (2002) discussed below. We calculated the ratio between the stress and the magnetic energy density associated with the primary MRI mode in two regimes of interest and obtained

$$
\begin{array}{ll}
\alpha_{\text {sat }} \beta_{\text {sat }} \simeq 0.4 \quad \text { for } \quad \Lambda_{\nu} \gg 1, \Lambda_{\eta}>1, \\
\alpha_{\text {sat }} \beta_{\text {sat }} \simeq 0.5 \Lambda_{\eta} \quad \text { for } \quad \Lambda_{\nu} \gg 1, \Lambda_{\eta}<1 .
\end{array}
$$

This anti-correlation between $\alpha_{\text {sat }}$ and $\beta_{\text {sat }}$ is seen in the numerical simulations of 'ideal' MHD (i.e., without explicit dissipation) in Hawley et al. (1995), as well as in the resistive runs in Sano et al. (1998). We understood these results in terms of the behavior of both primary and secondary modes.

We showed that important properties of the fastest secondary instabilities found in Pessah \& Goodman (2009) for the region of parameter space accessible to current numerical simulations are generic. The fastest parasitic modes are nonaxisymmetric, have purely real growth rates, have the same vertical periodicity as the primary MRI mode, and horizontal wavelengths that are roughly twice as long. Their wavevectors $\boldsymbol{k}_{\mathrm{h}}$ are nearly aligned with either the velocity or the magnetic field of the primary mode. The first type dominate for $\Lambda_{\eta} \geq 1$ and correspond to Kelvin-Helmholtz modes that feed off the velocity shear induced by the MRI and reach their maximum 
growth rates along the direction of the MRI velocity field. The second type dominate for $\Lambda_{\eta}<1$ and are related to tearing modes that feed off the MRI currents and grow the fastest along the direction of the MRI magnetic field.

We emphasized the importance of understanding the structure of the vorticity and current density patterns associated with the secondary instabilities, as they provide a mean to confirm their association with Kelvin-Helmholtz and tearing mode instabilities. The analysis presented in Section 6 suggests a strategy to recognize the presence of parasitic modes and attempt to identify their nature: (i) Evolve the simulation until the breakdown of the initial exponential growth or subsequent peaks in stress or magnetic energy. (ii) Dissect the simulation domain in planes perpendicular to the mid-plane that contain the $z$-axis. (iii) Project the velocity and magnetic field in these planes and take the curls in order to obtain the corresponding vorticity and current density. (iv) Determine whether these resemble what is expected from the KelvinHelmholtz or tearing mode instabilities.

\subsection{Connection to Previous Works}

\subsubsection{Evolution of MRI in Two and Three Dimensions, Extent of Simulation Domains, and Aspect Ratios}

Exploiting the numerical advantages of working with two-dimensional, axisymmetric simulations, Masada \& Sano (2008) explored the effects of explicit viscosity with $\Lambda_{\nu}=$ $\{0.01,0.1,1,10\}$ (and no explicit resistivity) and explicit resistivity with $\Lambda_{\eta}=\{0.1,0.3,1,10,100\}$ (and no explicit viscosity). The fact that the fastest secondary modes are not axisymmetric suggests that the transition to turbulence, and perhaps the subsequent non-linear evolution, should be rather different in two-dimensional, axisymmetric and three-dimensional numerical simulations, see e.g., Guan \& Gammie (2008); Obergaulinger et al. (2009). Indeed, the viscous simulations shown in Figures $4 a$ and 7 in Masada \& Sano (2008) do not seem to reach saturation.

The wavelength of the fastest growing MRI mode increases as viscosity and resistivity increase (Pessah \& Chan 2008). If the vertical extent of the simulation domain is not large enough, the fastest growing mode, or even the smallest unstable mode, might not fit in the domain. This might lead to spurious dependencies of the saturation on the dissipation coefficients (see below). Two- and three-dimensional simulations with aspect ratios $L_{r} / L_{z}=1$ seem to evolve differently than those with $L_{r} / L_{z} \simeq 2$. This has been seen in both types of setups by Obergaulinger et al. (2009) and Bodo et al. (2008). This could be due the fact that both axisymmetric and the fastest growing (non-axisymmetric) parasitic modes have horizontal wavelengths larger than the vertical wavelength of the primary MRI mode. However, once the fastest parasites are allowed to evolve unimpeded, increasing the aspect ratio further should not alter the results significantly.

Therefore, in order to reliably assess the dependence of the saturation of the MRI on the value of the dissipation coefficients, three-dimensional domains with $L_{z}>\lambda_{\mathrm{MRI}} \equiv$ $2 \pi / K_{\max }(\nu, \eta)$ and $L_{r} / L_{z}>2$ seem to be required.

\subsubsection{Transition to Turbulence and Recurrence of Channels}

As resistivity increases, the timescale for the MRI to grow to amplitudes such that the parasites become dynamically important also increases. This behavior is in agreement with the results presented in Fleming et al. (2000). Their Figure 2 shows that the mechanism that disrupts the initial exponential growth of the MRI (arguably related to the instabilities under study) is sensitive to the values of microphysical dissipation, with more resistive runs reaching higher amplitudes.

The ratio of the peak magnetic energy density between their more resistive runs, corresponding to $5 \Lambda_{\eta} \simeq 1$, and the least resistive simulation, which behaves similarly to their simulation without explicit dissipation, is about ten. This is in contrast to our expectation that these saturation amplitudes should not differ by more than a factor of two, see Figure 3 Note however, that the fastest growing mode in their 'ideal' run corresponds to $\lambda_{\mathrm{MRI}}=L_{z} / 2$ while in the very resistive runs $\lambda_{\mathrm{MRI}}=L_{z}$. This means that the fastest parasites, which quite generically posses horizontal wavelengths that are roughly a factor of two longer than the fastest growing MRI mode, might be suppressed in the more resistive simulations, which could account for the larger amplitudes observed.

Fleming et al. (2000) and Sano \& Inutsuka (2001) studied the effects of resistivity in the subsequent recurrent emergence of organized fluid motions with highly correlated magnetic fields (so called "channels"). The resistive simulations of Fleming et al. (2000) show the same type of quasi-periodic variations in the mean stress observed in 'ideal' MHD simulations after the break up of the initial exponential growth. The amplitude of the fluctuations around the mean and the timescales involved increase as resistivity increases, see their Figure 2. This type of fluctuations are known to be reduced significantly when the aspect ratio $L_{r} / L_{z}$ increases beyond a factor of two (Bodo et al. 2008). For the more resistive simulations, the wavelength of the fastest MRI mode is $\lambda_{\mathrm{MRI}}=L_{z}$. It is thus tempting to attribute the persistence of these fluctuations to the suppression of the fastest parasitic modes in the simulations with $L_{r} / L_{z}=1$.

The parasitic instabilities limit the amount of energy that the MRI can extract from the the differential rotation. This suggests that the larger peak amplitudes of the fluctuations in the more resistive runs could be due to the fact that the timescale for the MRI to grow to amplitudes such that the parasites become dynamically important increases with resistivity. Once the ordered motions of the primary modes are disrupted by the fastest available parasitic modes, non-linear interactions are arguably responsible for the cascade of energy to smaller scales where it dissipates. Since more magnetic energy needs to be dissipated after the reconnection of recurrent fluctuations with higher amplitudes, the longer timescales involved in the more resistive runs seems natural.

The fact that the peak amplitude of the initial exponential growth is larger than the subsequent fluctuations can be explained in terms of the corresponding amplitude of the seed fluctuations that excite the parasites. The amplitude to which the MRI can grow before the parasites reach similar amplitudes is sensitive to the initial amplitude of the seed fluctuations that excite the secondary instabilities. In the absence of explicit perturbations to seed the secondary instabilities in the linear regime of the MRI, the amplitude of the fastest growing MRI mode could overshoot our estimates by a large factor, which depends logarithmically on the amplitude of the seed fluctuations (Pessah \& Goodman 2009). The subsequent channels are emerging from a turbulent background where the seed fluctuations for the parasites is larger. Therefore these parasites will be able to reach an amplitude similar to that of the dominant primary MRI mode faster than the parasites

\footnotetext{
${ }^{5}$ Notice that their definition of magnetic Reynolds number $\mathrm{Rm}$ is related to our definition of Elsasser number via $\Lambda_{\eta} \equiv 2 \mathrm{Rm} / \beta$ with $\beta=400$.
} 
responsible for halting the initial exponential growth. This would result in smaller amplitudes for the subsequent channels (c.f. Latter et al. (2009), where it is suggested that the smaller amplitude of the recurrent channels is due to interactions between modes.)

There is numerical evidence that suggests that ohmic heating due to the reconnection of MRI field lines is an important source of energy in resistive MHD (Fleming et al. 2000; Sano \& Inutsuka 2001). Although this reconnection process has been attributed to the ideal parasitic instabilities studied in Goodman \& Xu (1994), this is a clear signature of nonideal MHD effects. In allowing for non-zero resistivity and calculating the currents associated with the secondary instabilities, we have gone one step forward in establishing the chain of processes that enable the conversion of gravitational energy into thermal energy in differentially rotating, magnetized, non-ideal plasmas. The properties of tearing modes discussed in Section 6 should provide better guidance for interpreting these reconnection events.

\subsubsection{Dependence of Saturation on Dissipation Coefficients: Elsasser Number vs. Magnetic Prandtl Number}

In the region of parameter space where our analysis overlaps with the regime accessible to the numerical simulations of Lesur \& Longaretti (2007), the values of $\alpha_{\text {sat }}$ are within factors of a few of the values obtained in the turbulent regime. The dependence on the stresses at saturation on the magnetic Prandtl number in our calculations is less pronounced than what they report and the predicted value of $\alpha_{\text {sat }}$ is smaller, by a factor of 6 at $\mathrm{Pm}=1$. Part of these differences can be accounted for with a more sensible operational definition of saturation. Pessah \& Goodman (2009) provide a way to estimate the amplitude of the fields at saturation when primary and secondary modes reach comparable amplitudes based on the values obtained when they reach equal growth rates. They conclude that the overshoot factor, which depends logarithmically on the initial amplitude of the parasite, is likely between $\simeq 3$ and $\simeq 10$. These arguments are applicable quite generically since they mainly rely on the fact that the growth rate of the fastest secondary modes is linear in the amplitude of the primary MRI mode, see Section 5 .

We posit that it is then conceivable that Equations (70) and (71) could provide, within factors of a few, a reasonable description of the saturation level of the MRI in a wide region of parameter space. Our results suggests that, as long as viscous dissipation does not dominate the dynamics of the fluid, i.e. $\Lambda_{\nu} \geq 10$, which is the case in many astrophysical environments, as well as in laboratory experiments, see Section 4 then the angular momentum transport due to the MRI depends mainly on the Elsasser number, see Figure 11 The numerical results presented in Sano \& Stone (2002) and Fleming et al. (2000) seem to support this statement.

Sano \& Stone (2002) carried out an extensive numerical study of the saturation of the MRI considering ohmic dissipation and Hall terms, with no explicit viscosity. They identify the existence of a critical Elsasser number of order unity, $\Lambda_{\eta}^{\mathrm{c}} \simeq 1$, which is independent of the strength and geometry of the magnetic field or the magnitude of the Hall term. For Elsasser numbers $\Lambda_{\eta}>1$, the stress at saturation is rather insensitive to $\Lambda_{\eta}$. However, for $\Lambda_{\eta}<1$, they find that the mean value of the stress decreases linearly with the Elsasser number, i.e., $\bar{T}_{r \phi}^{\text {sat }} \propto \Lambda_{\eta}$, see their Figure 20 . This result describes numerical simulations spanning a wide range of El- sasser numbers and magnetic field configurations. The inclusion of Hall terms does not seem to affect these results significantly. Even though the most resistive simulations carried out in Fleming et al. (2000) correspond to Elsasser numbers slightly larger than unity, their Table 1 shows that the stress at saturation decreases linearly with increasing Elsasser number.

Recent works investigating the effects of dissipation on the saturation of the MRI suggest that the saturation amplitude depends on viscosity and resistivity mainly through the magnetic Prandtl number, $\mathrm{Pm} \equiv \Lambda_{\eta} / \Lambda_{\nu}$. Umurhan et al. (2007a) performed a weakly non-linear analysis of the viscous, resistive MRI in the limit of small magnetic Prandtl number, i.e., $\mathrm{Pm} \ll 1$. They found that the saturation amplitude is proportional to $\mathrm{Pm}^{1 / 2}$, while the associated momentum transport scales as $\operatorname{Re}^{-1}$, where $\operatorname{Re} \equiv \Omega_{0} L^{2} / \nu$ is the Reynolds number. Lesur \& Longaretti (2007) carried out a series of shearing box simulations in incompressible MHD with explicit dissipation. In the range of parameters that they explored, i.e., $1 \lesssim \Lambda_{\nu} \lesssim 100$ and $0.1 \lesssim \mathrm{Pm} \lesssim 10$, the stress decreases with decreasing magnetic Prandtl number as $\mathrm{Pm}^{\delta}$ with $\delta \simeq 0.25-0.5$, with a weak dependence on the Reynolds number. Although it must be noted that the two approaches (weakly non-linear analysis vs. three-dimensional simulations) are indeed quite different, Lesur \& Longaretti (2007) suggest that the difference between their findings and those of Umurhan et al. (2007a) could be related to boundary conditions.

It seems reasonable to argue that the saturation of the MHD turbulence driven by the MRI at large values of magnetic Prandtl number with $\Lambda_{\eta}>\Lambda_{\nu} \gg 1$ should converge towards an asymptotic value. This limit does not seem to have been achieved in Figure 10 in Lesur \& Longaretti (2007) . Perhaps the highest Reynolds and magnetic Prandtl numbers considered, which correspond in our definitions to $\Lambda_{\nu} \simeq 10^{2}$ and $\mathrm{Pm} \simeq 10$, are not large enough to observe the asymptotic limit suggested by our analysis (see also their discussion regarding the effects of limited resolution for this case).

It is tempting to examine the seemingly discrepant dependencies of saturation on Elsasser and magnetic Prandtl number for the most dissipative simulations in Fleming et al. (2000) and Lesur \& Longaretti (2007). Fleming et al. (2000) considered ohmic dissipation but not explicit viscosity and it is thus hard to assign a well defined magnetic Prandtl number to their simulations. However, simulations without explicit dissipation seem to be characterized by an effective magnetic Prandtl number of order unity (Simon et al. 2009). We could speculate that the simulations with explicit resistivity but no explicit viscosity in Fleming et al. (2000) correspond to $\Lambda_{\eta}<\Lambda_{\nu}$, and thus the regime $\Lambda_{\eta} \simeq 1$ corresponds to $\mathrm{Pm} \lesssim 1$. However, the most inviscid and resistive simulations in Lesur \& Longaretti (2007) correspond to $\Lambda_{\nu} \simeq 10^{2}$ and $\mathrm{Pm} \simeq 0.1$, i.e., $\Lambda_{\eta} \simeq 10$. Thus, even though the viscosity seems small in these simulations, the Elsasser number does not seem to be low enough for the stress to show the behavior $\bar{T}_{r \phi}^{\text {sat }} \propto \Lambda_{\eta}$ for $\Lambda_{\eta}<1$ found in Sano \& Stone (2002).

Our results suggest that the Elsasser number dictates the

${ }^{6}$ Notice that the subset of simulations with vertical background fields in Sano \& Stone (2002), show a dependence with $\Lambda_{\eta}$ that is steeper than linear. The inspection of their Table 1 suggests that this could be due to: (i) resolution constrains, for the simulations where several fast growing MRI modes fit within the box, (ii) aspect ratios constrains, for the simulations where the most unstable mode is well resolved but its vertical wavelength is of the order of $L_{z}$, or (iii) because the most unstable mode does not fit within the box. 
saturation level of the angular momentum transport driven by MHD turbulence in astrophysical disks and experiments, see Figure 11. Despite the inherent limitations of the parasitic mode analysis presented here, this result seems to be supported by numerical simulations of Fleming et al. (2000) and Sano \& Stone (2002). However, given the rather small dynamical range of dissipation coefficients that can be currently explored, these conclusions warrant further examination. Additional numerical studies with explicit viscosity and resistivity in three-dimensional domains (that can accommodate for the most relevant MRI and parasitic modes) seem to be necessary to fully address whether the main parameter determining the saturation of the MRI is the Elsasser number or the mag- netic Prandtl number.

I am grateful to Jeremy Goodman for his encouragement and many insightful conversations. I thank Chi-kwan Chan, Peter Goldreich, Yoram Lithwick, Aldo Serenelli, and Jihad Touma for useful discussion throughout this study. I thank the anonymous referee for carefully reading the original manuscript and providing comments that helped improve this paper. The calculations presented in this paper were carried out in the Aurora Cluster at the Institute for Advanced Study. I gratefully acknowledge the W. M. Keck Foundation and the Institute for Advanced Study for their generous support.

\section{REFERENCES}

Arnett, D., Meakin, C., \& Young, P. A. 2009, ApJ, 690, 1715

Balbus, S. A., \& Hawley, J. F. 1991, ApJ, 376, 214 1998, Rev. Mod. Phys., 70, 1

Balbus, S. A., \& Henri, P. 2008, ApJ, 674, 408

Batchelor, G. K. 2000, An Introduction to Fluid Dynamics (Cambridge: Cambridge Univ. press)

Blackman, E. G., Penna, R. F., \& Varnière, P. 2008, New Astron., 13, 244

Brandenburg, A., Nordlund, A., Stein, R. F., \& Torkelsson, U. 1995, ApJ, 446,741

Brandenburg, A., \& Subramanian, K., 2005, PhR, 417, 1

Bodo, G., Mignone, A., Cattaneo, F., Rossi, P., \& Ferrari, A. 2008, A\&A, 487,1

Boyd, T. J. M., \& Sanderson, J. J. 2003, The Physics of Plasmas (Cambridge: Cambridge University Press)

Chandrasekhar, S. 1960, Proc. Nat. Acad. Sci., 46, 253

Fleming, T. P., Stone, J. M., \& Hawley, J. F. 2000, ApJ, 530, 464

Frank, J., King, A., \& Raine, D. J. 2002, Accretion Power in Astrophysics (3rd ed.; Cambridge: Cambridge Univ. Press)

Fromang, S., Papaloizou, J., Lesur, G., \& Heinemann, T. 2007, A\&A, 476, 1123

Furth, H. P., Killeen, J. \& Rosenbluth, M. N. 1963, PhFl, 6, 459

Gammie, C. F. 1996, ApJ, 462, 725

Goodman, J., \& Ji, H. 2002, JFM, 462, 365

Goodman, J., \& Xu, G. 1994, ApJ, 432, 213

Guan, X. \& Gammie, C. F. 2008, ApJS, 174, 145

Hawley, J. F., Gammie, C. F., \& Balbus, S. A. 1995, ApJ, 440, 742

Hubbard, A., \& Blackman, E. G. 2008, MNRAS, 390, 331

Jamroz, B., Julien, K., \& Knobloch, E. 2008, AN, 329, 675

Jamroz, B., Julien, K., \& Knobloch, E. 2008, PhST, 132, 4027

Ji, H., Goodman, J., \& Kageyama, A. 2001, MNRAS, 325, L1

Jin, L. 1996, ApJ, 457, 798

Kato, S., \& Yoshizawa, A. 1995, PASJ, 47, 629

Knobloch, E., \& Julien, K. 2005, Phys. Fluids, 17, 094106

Latter, H. N., Lesaffre, P., \& Balbus, S. A. 2009, MNRAS, 394, 715

Lesaffre, P., Balbus, S. A., \& Latter, H. 2009, MNRAS, 396, 779

Lesur, G., \& Longaretti, P. Y. 2007, MNRAS, 378, 1471

Liu, W., Goodman, J. \& Ji, H. 2006, ApJ, 643, 306

Lynden-Bell, D., \& Pringle, J. E. 1974, MNRAS, 168, 603
Masada, Y., \& Sano, T. 2008, ApJ, 689, 1234

Miller, K. A., \& Stone, J. M. 2000, ApJ, 534, 398

Nornberg, M. D., Ji, H., Schartman, E., Roach, A. \& Goodman, J. 2010, Phys. Rev. Lett. 104, 074501

Obergaulinger, M., Cerdá-Durán, P., Müller, E., \& Aloy, M. A. 2009, A\&A, 498, 241

Ogilvie, G. I. 2003, MNRAS, 340, 969

Pessah, M. E., \& Chan, C. K. 2008, ApJ, 684, 498

Pessah, M. E., Chan, C. K., \& Psaltis, D. 2006a, MNRAS, 372, 183

Pessah, M. E., Chan, C. K., \& Psaltis, D. 2006b, Phys. Rev. Lett., 97, 221113

Pessah, M. E., Chan, C. K., \& Psaltis, D. 2007, ApJ, 668, L51

Pessah, M. E., Chan, C. K., \& Psaltis, D. 2008, MNRAS, 383, 683

Pessah, M. E., \& Goodman, J. 2009, ApJ, 698, L72

Rüdiger, G., Schultz, M., \& Shalybkov, D. 2003, Phys. Rev. E, 67, 046312

Salmeron, R.\& Wardle, M. 2005, MNRAS, 361, 45

Sano, T. 2007, Ap\&SS, 307, 191

Sano, T., \&, Inutsuka, S. I. 2001, ApJ, 561, L179

Sano, T., Inutsuka, S. I., \& Miyama, S. M. 1998, ApJ, 506, L57

Sano, T., Inutsuka, S. I., Turner, N. J., \& Stone, J. M. 2004, ApJ, 605, 321

Sano, T., \& Miyama, S. M. 1999, ApJ, 515, 776

Sano, T., \& Stone, J. M. 2002, ApJ, 577, 534

Shakura, N. I., \& Sunyaev, R. A. 1973, A\&A, 24, 337

Simon, J. B., Hawley, J. F., \& Beckwith, K. 2009, ApJ, 690, 974

Sisan, D. R. Mujica, N., Tillotson, W. A., Huang, Y. M., Dorland, W., Hassam, A. B., Antonsen, T. M., \& Lathrop, D. P. 2004, Phys. Rev. Lett., 93, 114502

Sturrock, P. A. 1994, Plasma Physics: an Introduction to the theory of Astrophysical, Geophysical, and Laboratory Plasmas (Cambridge: Cambridge Univ. Press)

Tatsuno T., \& Dorland W. 2008, Astron. Nachr., 329, 688

Turner, N. J., Sano, T. \& Dziourkevitch, N. 2007, ApJ, 659, 729

Umurhan, O. M., Menou, K., \& Regev, O. 2007, Phys. Rev. Lett., 98 , 034501

Umurhan, O. M., Regev, O., \& Menou, K. 2007, Phys. Rev. E, 76, 6310

Velikhov, E. P. 1959, JETP, 36, 1398

Vishniac, E. 2009, ApJ, 696, 1021 